\title{
Numerical Prediction of Turbulent Convective Heat Transfer in Mini/Micro Channels for Carbon Dioxide at Supercritical Pressure
}

\author{
Pietro Asinari \\ Dipartimento di Energetica, Politecnico di Torino \\ Corso Duca degli Abruzzi 24, Zip Code 10129, Torino, Italy \\ Tel. +39-011-564-4413, Telefax. +39-011-564-4499
}

\begin{abstract}
A new approach to take into account the effects of variable physical properties on turbulence is suggested. It allows to choose freely the turbulent closure model for conventional terms due to velocity fluctuations and to describe coherently the additional terms due to density fluctuations. Numerical calculations based on the suggested approach have been performed for carbon dioxide flowing within mini/micro channels under cooling conditions. The numerical predictions show that the effects due to density fluctuations are smaller than it could have been initially supposed and that the heat transfer impairment for mini/micro channels, which some experiments seem to highlight, is not completely explained by the considered model.
\end{abstract}

Key words: Forced convection, Supercritical, Density Fluctuations, Turbulence Models, Cooling, Carbon Dioxide

Email address: pietro.asinari@polito.it (Pietro Asinari). 


\section{Nomenclature}

A surface $\left[m^{2}\right]$

$b$ dimensionless factor $[-]$

Bo buoyancy parameter [-]

$c_{p} \quad$ specific heat capacity $\left[J k^{-1} K^{-1}\right]$

$C$ robust correlation coefficient [-]

$d \quad$ diameter of mini/micro channel $[\mathrm{m}]$

$e \quad$ dimensionless error $[-]$

f generic thermophysical properties

F corrective tensor due to density fluctuations [-]

$g \quad$ acceleration due to gravity $\left[\mathrm{ms}^{-2}\right]$

$G \quad$ mass flow rate $\left[\mathrm{kg} \mathrm{s}^{-1}\right]$

Gr Grashof number [-]

$h \quad$ specific enthalpy $\left[\mathrm{J} \mathrm{kg}^{-1}\right]$

$H$ generic source term

I identity matrix [-]

$k \quad$ turbulent kinetic energy $\left[\mathrm{J} \mathrm{kg}^{-1}\right]$

$L \quad$ length of the mini/micro channel $[\mathrm{m}]$

$M \quad$ identifier of turbulent closure model

$N \quad$ natural number

$\mathcal{N}^{+}$set of positive natural numbers

$p \quad$ pressure $[P a]$

$\operatorname{Pr} \quad$ Prandtl number [-]

$q \quad$ thermal flux $\left[W m^{-2}\right]$

$\mathcal{Q} \quad$ set of fractional numbers

$r$ radial coordinate $[m]$

$\mathcal{R}$ set of real numbers 
Re Reynolds number [-]

$\mathbf{S} \quad$ stress tensor $\left[\mathrm{N} \mathrm{m}^{-2}\right]$

$T$ temperature $[K]$

$u \quad$ velocity component along axial direction $\left[\mathrm{ms}^{-1}\right]$

$v \quad$ velocity component along radial direction $\left[\mathrm{m} \mathrm{s}^{-1}\right]$

$V \quad$ volume $\left[\mathrm{m}^{3}\right]$

w velocity vector $\left[\mathrm{ms}^{-1}\right]$

$x \quad$ axial coordinate $[m]$

$y \quad$ distance from the wall $[m]$

z generic quantity

Greek symbols

$\alpha \quad$ convective heat transfer coefficient $\left[W m^{-2} K^{-1}\right]$

$\beta \quad$ modified compressibility factor $\left[\mathrm{kg} \mathrm{J}^{-1}\right]$

$\delta \quad$ Kroneker's symbol

$\epsilon \quad$ turbulent dissipation rate $\left[\mathrm{m}^{2} \mathrm{~s}^{-3}\right]$

$\phi \quad$ correction factor due to density fluctuations [-]

$\varphi$ non-ideal gas parameter $[-]$

$\Phi$ rounding function

$\lambda$ thermal conductivity $\left[W m^{-1} K^{-1}\right]$

$\mu \quad$ dynamic viscosity $\left[N s m^{-2}\right]$

$\eta$ radial location of pseudo-critical temperature $[m]$

$\omega$ generic solving variable

$\Omega$ computational domain

$\rho \quad$ density $\left[\mathrm{kg} \mathrm{m}^{-3}\right]$

$\sigma \quad$ intensity index $\left[\mathrm{J} \mathrm{kg}^{-1}\right]$

$\theta \quad$ temperature difference $[K]$

$\chi$ ratio of geometric progression $[-]$

$\zeta$ sign of enthalpy gradient $[-]$ 
Subscripts and superscripts

$\begin{array}{ll}a & \text { axial/center-line condition } \\ b & \text { bulk condition } \\ B R & \text { Bellmore and Reid } \\ c & \text { critical condition } \\ \text { exp } & \text { experimental condition } \\ l & \text { laminar condition } \\ L & \text { referring to whole length of mini/micro channel } \\ \lambda & \text { referring to thermal conductivity } \\ m & \text { minimum value } \\ \mu & \text { referring to dynamic viscosity } \\ R N G & \text { RNG } k-\epsilon \text { model } \\ S K E & \text { standard } k-\epsilon \text { model } \\ S, N, E, W & \text { cardinal directions } \\ t & \text { turbulent condition } \\ T & \text { total/stagnation condition } \\ w & \text { wall condition }\end{array}$

Notation

$\partial_{x} \quad$ partial derivative along axial direction $=\partial / \partial x$

$\partial_{r} \quad$ partial derivative along radial direction $=\partial / \partial r$

$\mathbf{z}_{1} \otimes \mathbf{z}_{2} \quad$ diadic product

$\langle z\rangle \equiv \bar{z} \quad$ time averaging 


\section{Introduction}

Turbulent convective heat transfer in ducts at supercritical pressure is encountered in a wide variety of engineering situations. Only some of them involve strong dependence of thermodynamic and transport properties on temperature that occurs near the critical point. In these cases, strong coupling between energy and momentum equation gives rise to unconventional effects, which are defined as critical phenomena. Some engineering applications which involve critical phenomena are: supercritical extraction; enhanced oil recovery; supercritical pollution oxidation; transcritical refrigerating cycles and supercritical coolants. In particular, recent researches in refrigeration technology have produced many experimental data for heat transfer near the critical point.

Increasing attention to environmental issues induces to reconsider natural fluids as alternative refrigerants. The carbon dioxide is a non-flammable and non-toxic fluid, which represents the primary candidate for air-conditioning with natural fluid [1]. Since in common refrigerating cycles, the heat rejection temperatures are usually above the critical temperature of carbon dioxide $\left(T_{c}=304.13 \mathrm{~K}\right)$, the heat rejection process usually occurs at a supercritical pressure $\left(p_{c}=7.377 \mathrm{MPa}\right)$ in order to maximize the cooling capacity. The high working pressure and the favorable heat transfer properties of carbon dioxide allow to use extruded flat tubes with circular/elliptical ducts, which have diameters much smaller than usual ducts $(d<2 \mathrm{~mm})$ [2]. Size reduction justifies the conventional name of mini/micro channels. Inside each mini/micro channel, the gas cooling process takes place without phase change, since the working fluid is at a supercritical pressure. In order to design a highefficiency heat exchanger for heat rejection, a deep understanding of turbulent convective heat transfer for supercritical carbon dioxide in cooled mini/micro channels is needed.

The highest temperature at which condensation/evaporation occurs is known as the critical temperature. Both theoretical and experimental evidences exist which indicate that the idea of a definite critical point, with unambiguous critical temperature, pressure and volume is probably only an approximation; actually there appears to be a critical region [4]. In this critical region the thermophysical properties have a strong dependence on temperature. For each supercritical pressure, the value of temperature at which the specific heat reaches a peak is called pseudo-critical temperature $T_{p c}$. At the pseudo-critical temperature, the thermal conductivity shows a weaker peak too. When the bulk temperature decreases below the pseudo-critical temperature for the considered supercritical pressure, the fluid instantaneously changes from a gas-like state to a liquid-like state [3]. In particular both the density and dynamic viscosity abruptly increase, in order to match the liquid-like behavior. As a result of the strong dependence of physical properties on temperature, convective heat transfer at supercritical pressure is generally more complex than commmon applications. High specific heat, significant thermal expansion, enhanced 
buoyancy and wall variations of thermal conductivity can produce important influences on convection [5]. Moreover if the physical properties change with temperature, it becomes necessary to take into account the influence of variable physical properties on the turbulent diffusivity expressions [6].

The previous effects probably justify the great discrepancies among different phenomenological correlations, which have been suggested throughout the last years. A comprehensive review of the heat transfer and pressure drop characteristics can be found in a specific paper [7]. In the following the main results will be summarized and recent developments, not included in cited paper, will be discussed. Initially only few correlations were specifically developed to calculate the heat transfer coefficients during cooling configurations near the critical point. Krasnoshchekov et al. [8] carried out the first experimental study on the heat transfer characteristics during turbulent flow in a horizontal tube with carbon dioxide at supercritical pressure under cooling conditions. Baskov et al. [9] found that their measurements for vertical tube were systematically lower than those calculated using the previous formula. They excluded the effect of buoyancy by comparing the results for ascending and descending tubes and suggested a new improved correlation. Petrov and Popov [10] numerically developed a correlation for configurations where free convection is negligible and found good agreement with experimental data. More recently, Pettersen et al. [11] experimentally found for extruded flat tubes with mini/micro channels and carbon dioxide at supercritical pressure that a usual correlation, originally developed for constant properties, can be suitably applied. Pitla et al. [12] suggested to improve this conventional correlation by averaging the results obtained with constant properties evaluated at the wall and bulk temperature. Finally, Yoon et al. [15] found that all previous studies generally under-predict their measures and proposed a new phenomenological correlation, which adopts the same functional dependence originally proposed by Krasnoshchekov et al. [8]. This brief review clearly shows a circular nature. Moreover only Liao and Zhao [13] investigated a single horizontal mini/micro channel with supercritical carbon dioxide and found that size reduction causes a heat transfer impairment, which cannot be predicted by correlations developed for usual ducts. Liao and Zhao [13] measured the variation of Nusselt number $N u_{b}$ with the bulk mean temperature for various tube diameters, keeping fixed the Reynolds number $R e_{b}$, the Prandtl number $\mathrm{Pr}_{b}$ and the difference between the bulk and wall temperature. The Nusselt number was found to decrease as the tube size became smaller and this means that a heat transfer impairment due to size reduction could exist. Liao and Zhao pointed out that buoyancy effect could be responsible of this phenomenon. Since the density considerably changes with temperature near the critical point, free convection can influence heat transfer in supercritical forced flow too. Theoretical considerations lead to the following criterion for 
negligible buoyancy effects in horizontal tubes [14]:

$$
\frac{G r}{R e_{b}^{2}}=\left(\frac{\rho_{w}}{\rho_{b}}-1\right) \frac{g d}{u_{b}^{2}}<1 \times 10^{-3}
$$

The Grashof number $\mathrm{Gr}$ represents the relative strength of secondary flow induced by the buoyancy force. Considering that the buoyancy parameter $G r / R e_{b}^{2}$ is proportional to the tube diameter $d$, for each operative configuration a critical diameter exists and all tubes characterized by smaller diameters are free of buoyancy effects. Liao and Zhao found that, for their experimental tests, this critical diameter is comparable to the upper diameter, which identifies the conventional class of mini/micro channels. They conclude that the heat transfer impairment could be caused, partially at least, by the fact that the buoyancy effect becomes less important for small tubes. In particular, in the region near the pseudo-critical temperature, the experimental data show wall thermal fluxes much lower than those predicted by correlation of Petrov and Popov [10]. In spite of the fact that the buoyancy effect was not included in the original correlation of Petrov and Popov, Liao and Zhao suggest that the correlation fails when free convection becomes weak or absent, because it was developed based on data for large-diameter tubes where this effect should be significant.

This explanation is not completely satisfactory. For horizontal tubes, buoyancy causes circumferential variations of heat transfer. When buoyancy effects are relevant, the upper part of the tube is characterized by impaired heat transfer while the lower one by enhanced heat transfer, both because of stratification of the flow [5]. The small amount of available data does not allow to predict clearly the net results of the two circumferential parts with regards of total heat transfer. Some evidences exist which buoyancy reduces the total heat transfer in horizontal tubes, though not in a very pronounced manner [6]. Firstly if tested mini/micro channels are characterized by negligible buoyancy effects, a small increase of heat transfer should be expected comparing with large-diameter tubes, contrary to experimental data. Secondly the correlation of Petrov and Popov does not take into account buoyancy effects. In a preliminary work, Petrov and Popov [16] solved numerically a system of equations which included also buoyancy in order to reproduce the experimental results of Baskov et al. [9]. The numerical calculations confirmed that the natural convection only slightly affects the heat transfer for the ranges of parameters considered in this experiment. For this reason, in the original where their correlation was suggested, the buoyancy was dropped out from the system of equations and no buoyancy parameter was included in the interpolation formula [10]. The effect of free convection was considered only in a following paper [17].

According to experimental data, mini/micro channels for considered conditions reveal a peculiar behavior in comparison with large-diameter tubes, i.e. 
heat transfer impairment, which has not been completely explained. Numerical investigation is an useful tool to test additional effects which could explain heat transfer impairment by overcoming some experimental difficulties. On the track of the work of Petrov and Popov, the present paper aims to investigate numerically the turbulent convective heat transfer in mini/micro channels for carbon dioxide at supercritical pressure. A new approach to take into account the effects of variable physical properties on turbulence is suggested, in order to widen the available numerical tools. Three numerical models are solved on a meaningful experimental plan in order to test their suitability to explain heat transfer impairment in considered conditions. Finally, a comparison with phenomenological correlations developed for usual ducts is also reported.

\section{Physical models}

Since the explanation of Liao and Zhao for heat transfer impairment lies in the fact that buoyancy is negligible for some working conditions of mini/micro channels, in the following only pure forced convective regime will be considered. This means that the limiting condition (1) is punctually verified. If the gravitational field is neglected, the problem of turbulent convective heat transfer inside horizontal circular tubes shows no angular dependence.

Because of size reduction, the ratio between surface roughness and characteristic diameter increases. Topology of mini/micro channel inner surface becomes more important at smaller diameters and could affect heat transfer too. Experimental data for aluminum mini/micro channels, with the smallest diameter considered in the following, show only negligible discrepancies $(6 \%)$ between measured pressure drops and Blasius's correlation, which was developed for hydraulically smooth regime [11]. It is reasonable to suppose that stainless steel mini/micro channels considered by Liao and Zhao were characterized by lower roughness. This hypothesis is confirmed by the fact that Liao and Zhao correlation is a modified version of Dittus-Boelter correlation, which does not take into account roughness [13]. Finally, if a roughness effect exists, the analogy between fluid flow and heat transfer induces to suppose that it should enhance heat transfer, contrary to experimental evidence. In the following the mini/micro channel will be considered hydraulically smooth over the entire investigated range of Reynolds number.

Turbulent forced convection heat transfer is described by the instantaneous conservation equations of continuity, momentum and energy. When the physical properties rapidly change with temperature, as it happens near the critical point, turbulent regime is characterized by high-frequency fluctuations of physical properties, in addition to common fluctuations of velocity components and temperature. Reynolds averaging, i.e. time averaging, of governing equations produces additional unknown quantities, which must be calculated in terms of 
solving variables. In particular, effects due to density are stronger than those due to diffusivities, such as dynamic viscosity and thermal conductivity [18]. For this reason, Favre averaging, i.e. density-weighted averaging, appears more suitable because density fluctuations are automatically taken into account by averaging procedure [7]. The problem is only apparently simplified because, in the final system of equations, the density-averaged quantities appear, which differ from common time-averaged ones. In particular, the phenomenological coefficients of turbulent closure models come from an empirical fitting of time-averaged measures and they are inapplicable to reduced turbulent quantities due to Favre averaging. Although superior in principle, in this work the density-weighted averaging will be abandoned for more usual time-averaging, essentially because extensive validation data are lacking.

On introducing the Reynolds decomposition for velocity $\mathbf{w}=\overline{\mathbf{w}}+\mathbf{w}^{\prime}$ and density $\rho=\bar{\rho}+\rho^{\prime}$ into instantaneous conservation equations and time-averaging the results, the governing equations of continuity, momentum and energy are obtained.

$$
\begin{aligned}
& \nabla \cdot\left[\bar{\rho}\left(\overline{\mathbf{w}}+\overline{\mathbf{w}}^{*}\right)\right]=0 \\
& \nabla \cdot\left[\bar{\rho} \overline{\mathbf{w}} \otimes\left(\overline{\mathbf{w}}+\overline{\mathbf{w}}^{*}\right)\right]=-\nabla p+\nabla \cdot \mathbf{S} \\
& \nabla \cdot\left[\bar{\rho} \overline{h_{T}}\left(\overline{\mathbf{w}}+\overline{\mathbf{w}}^{*}\right)\right]=-\nabla \cdot \mathbf{q}+\nabla \cdot(\mathbf{S} \overline{\mathbf{w}})
\end{aligned}
$$

where $\overline{\mathbf{w}}^{*}=\overline{\rho^{\prime} \mathbf{w}^{\prime}} / \bar{\rho}$ is the characteristic velocity for density fluctuations, $\mathbf{S}=\mathbf{S}^{l}+\mathbf{S}^{t}$ is the effective stress tensor and $\mathbf{q}=\mathbf{q}^{l}+\mathbf{q}^{t}$ is the effective thermal flux. Laminar and turbulent components for both effective stress tensor and effective thermal flux are defined as follow.

$$
\begin{aligned}
& \mathbf{S}^{l}=\mu\left(\nabla \overline{\mathbf{w}}+\nabla \overline{\mathbf{w}}^{T}\right)-(2 / 3 \mu \nabla \cdot \overline{\mathbf{w}}) \mathbf{I} \\
& \mathbf{S}^{t}=-\bar{\rho} \overline{\mathbf{w}^{\prime} \otimes \mathbf{w}^{\prime}}-\overline{\rho^{\prime} \mathbf{w}^{\prime}} \otimes \overline{\mathbf{w}}-\overline{\rho^{\prime} \mathbf{w}^{\prime} \otimes \mathbf{w}^{\prime}} \\
& \mathbf{q}^{l}=-\lambda \nabla T \\
& \mathbf{q}^{t}=\bar{\rho} \overline{h_{T}^{\prime} \mathbf{w}^{\prime}}+\overline{\rho^{\prime} h_{T}^{\prime}} \overline{\mathbf{w}}+\overline{\rho^{\prime} h_{T}^{\prime} \mathbf{w}^{\prime}}
\end{aligned}
$$

The last equation can be easily simplified by considering that $\overline{h_{T}^{\prime} z^{\prime}}-\overline{h^{\prime} z^{\prime}}=$ $1 / 2 \overline{\mathbf{w}^{\prime} \cdot \mathbf{w}^{\prime} z^{\prime}}<<\overline{h^{\prime} z^{\prime}}$, where $z$ can be indifferently $\rho, u$ or $v$. Some of the previous terms due to turbulent fluctuations can be expressed by means of turbulent-viscosity hypothesis and gradient-diffusion hypothesis [19].

$$
\begin{aligned}
& -\bar{\rho} \overline{\mathbf{w}^{\prime} \otimes \mathbf{w}^{\prime}}=\mu_{t} / \mu \mathbf{S}^{l} \\
& \bar{\rho} \overline{h^{\prime} \mathbf{w}^{\prime}}=\lambda_{t} / \lambda \mathbf{q}^{l}
\end{aligned}
$$


A tensor $\mathbf{F}^{\mu}$ can be introduced to describe the effects due to density fluctuations on effective stress tensor $\mathbf{S}=\left(\mathbf{I}+\mu_{t} / \mu \mathbf{F}^{\mu}\right) \mathbf{S}^{l}$. In the same way, a tensor $\mathbf{F}^{\lambda}$ can be introduced to describe the effects due to density fluctuations on effective thermal flux $\mathbf{q}=\left(\mathbf{I}+\lambda_{t} / \lambda \mathbf{F}^{\lambda}\right) \mathbf{q}^{l}$.

$$
\begin{aligned}
& \mathbf{F}^{\mu}=\mathbf{I}+\left(\overline{\rho^{\prime} \mathbf{w}^{\prime}} \otimes \overline{\mathbf{w}}+\overline{\rho^{\prime} \mathbf{w}^{\prime} \otimes \mathbf{w}^{\prime}}\right)\left(\bar{\rho} \overline{\mathbf{w}^{\prime} \otimes \mathbf{w}^{\prime}}\right)^{-1} \\
& \mathbf{F}^{\lambda}=\mathbf{I}+\left(\overline{\rho^{\prime} h^{\prime}} \overline{\mathbf{w}}+\overline{\rho^{\prime} h^{\prime} \mathbf{w}^{\prime}}\right) \otimes \overline{h^{\prime} \mathbf{w}^{\prime}} /\left(\bar{\rho} \overline{h^{\prime} \mathbf{w}^{\prime}} \cdot \overline{h^{\prime} \mathbf{w}^{\prime}}\right)
\end{aligned}
$$

In particular, density fluctuations affect both the diffusive and the convective terms into equations $(2,3,4)$. Since $\mathbf{F}^{\mu}$ is not symmetric, then effective stress tensor $\mathbf{S}$ is not simmetric too.

Keeping in mind the geometrical configuration realized by mini/micro channels, a two dimensional computational domain $\Omega \in \mathcal{R}^{2}$ will be considered and a set of cylindrical coordinates will be adopted to describe it $\Omega=\{(x, r) \in$ $\left.\mathcal{R}^{2}: 0 \leq x \leq L, 0 \leq r \leq R\right\}$. The components of velocity vector will be accordingly renamed $\overline{\mathbf{w}}=(\bar{u}, \bar{v})$. Because of the simplifying assumptions in boundary layer theory [20], the momentum and energy equations can be simplified to yield approximate solutions for this case.

$$
\begin{aligned}
& \nabla_{x r} \cdot\left[\bar{\rho}\left(\overline{\mathbf{w}}+\overline{\mathbf{w}}^{*}\right)\right]=0 \\
& \nabla_{x r} \cdot\left[\bar{\rho} \bar{u}\left(\overline{\mathbf{w}}+\overline{\mathbf{w}}^{*}\right)\right]=-\frac{d p}{d x}+\frac{1}{r} \frac{\partial}{\partial r}\left(r S_{x r}\right) \\
& \nabla_{x r} \cdot\left[\bar{\rho} \overline{h_{T}}\left(\overline{\mathbf{w}}+\overline{\mathbf{w}}^{*}\right)\right]=+\frac{1}{r} \frac{\partial}{\partial r}\left(r \bar{u} S_{r x}-r q_{r}\right)
\end{aligned}
$$

Since the laminar stress tensor reduces to $S_{i j}^{l}=\mu \partial_{r} \bar{u}\left(1-\delta_{i j}\right)$, then all the components of effective stress tensor can be expressed in terms of transverse velocity gradient $S_{i j}=\left[\mu\left(1-\delta_{i j}\right)+\mu_{t} F_{i k}^{\mu}\left(1-\delta_{k j}\right)\right] \partial_{r} \bar{u}$. In the same way, the concept of thermal boundary layer simplifies the calculation of thermal fluxes. Since the laminar thermal flux reduces to $q_{i}^{l}=\lambda \partial_{r} T\left(1-\delta_{i 1}\right)$, then all the components of effective thermal flux can be expressed in terms of transverse temperature gradient $q_{i}=\left[\lambda\left(1-\delta_{i 1}\right)+\lambda_{t} F_{i k}^{\lambda}\left(1-\delta_{k 1}\right)\right] \partial_{r} T$. In the following some components of the tensors, which describe density fluctuations, are reported, because they are involved in the calculation of effective diffusive terms $S_{x r}, S_{r x}$ and $q_{r}$ in simplified equations $(14,15)$.

$$
\begin{aligned}
& F_{x x}^{\mu}=1+\frac{\overline{\rho^{\prime} u^{\prime}} \bar{v}}{\bar{\rho} \overline{u^{\prime} v^{\prime}}}+\frac{\overline{\rho^{\prime} u^{\prime} v^{\prime}}}{\bar{\rho} \overline{u^{\prime} v^{\prime}}} \\
& F_{r r}^{\mu}=1+\frac{\overline{\rho^{\prime} v^{\prime}} \bar{u}}{\bar{\rho} \overline{u^{\prime} v^{\prime}}}+\frac{\overline{\rho^{\prime} u^{\prime} v^{\prime}}}{\bar{\rho} \overline{u^{\prime} v^{\prime}}}
\end{aligned}
$$




$$
F_{r r}^{\lambda}=1+\frac{\overline{\rho^{\prime} h^{\prime}} \bar{v}}{\bar{\rho} \overline{h^{\prime} v^{\prime}}}+\frac{\overline{\rho^{\prime} h^{\prime} v^{\prime}}}{\bar{\rho} \overline{h^{\prime} v^{\prime}}}
$$

The residual components of effective stress tensor may differ $S_{x r} \neq S_{r x}$ because they involve different components of tensor which describes density fluctuations $F_{x x}^{\mu} \neq F_{r r}^{\mu}$. The term $F_{x x}^{\mu}$ directly affects the turbulent viscosity, while the term $F_{r r}^{\mu}$ describes the effect of density fluctuations on viscous heating and it can be usually neglected. For this reason, all considered models assume $F_{r r}^{\mu} \approx 1$ and consequently $S_{r x} \approx\left(1+\mu_{t} / \mu\right) S_{r x}^{l}=\left(1+\mu_{t} / \mu\right) S_{x r}^{l}$. Moreover density fluctuations are differently involved into convection along main directions. In order to estimate this discrepancy, an auxiliary radial velocity $\bar{v}_{0}$ is introduced, which represents the radial velocity field obtained neglecting the density fluctuations. Assuming fixed the mean density distribution, this auxiliary function satisfies the following equation.

$$
\frac{\partial}{\partial x}(\bar{\rho} \bar{u})+\frac{1}{r} \frac{\partial}{\partial r}\left(r \bar{\rho} \bar{v}_{0}\right)=0
$$

Along axial direction, the effect of density fluctuations can be clearly neglected $\left|\bar{u}^{*}\right|<<|\bar{u}|$. Along radial direction, considering equations $(13,19)$ and applying proper boundary conditions, a relation among actual, characteristic and auxiliary radial velocities can be found $\bar{v}+\bar{v}^{*}=\bar{v}_{0}$. In the region near the critical point, strong density fluctuations ensure $\left|\bar{v}^{*}\right|>>\left|\bar{v}_{0}\right|$. For all the following calculations, this condition has been verified for at least one order of magnitude. In this case, an easy correlation yields $\bar{v}+\bar{v}^{*} \approx 0$ and it can be applied to simplify expressions $(16,18)$. Finally, the density fluctuations must be related to common fluctuations involved into turbulent closure models. The key idea is to expand the equation of state $\rho(h, p)$ by considering fluctuations of independent variables $\rho-\bar{\rho}=\left.\partial_{h} \bar{\rho}\right|_{p}(h-\bar{h})+\left.\partial_{p} \bar{\rho}\right|_{h}(p-\bar{p})$. Neglecting pressure variations, the residual term can be expressed by means of a modified compressibility $\beta=-\left.\partial_{h} \bar{\rho}\right|_{p} / \bar{\rho}$, in order to find the final correlation $\rho^{\prime}=-\bar{\rho} \beta h^{\prime}$. This correlation can be applied into the definition of characteristic velocity due to density fluctuations $\overline{\mathbf{w}}^{*}=-\beta \overline{h^{\prime} \mathbf{w}^{\prime}}$, which influences the convective terms, and into the definitions of correction factors $F_{x x}^{\mu}$ and $F_{r r}^{\lambda}$, which influence the diffusive terms.

$$
\begin{aligned}
& F_{x x}^{\mu}=1-\beta^{2} \frac{\overline{h^{\prime} u^{\prime}} \overline{h^{\prime} v^{\prime}}}{\overline{u^{\prime} v^{\prime}}}-\beta \frac{\overline{h^{\prime} u^{\prime} v^{\prime}}}{\overline{u^{\prime} v^{\prime}}} \\
& F_{r r}^{\lambda}=1-\beta^{2} \overline{h^{\prime} h^{\prime}}-\beta \frac{\overline{h^{\prime} h^{\prime} v^{\prime}}}{\overline{h^{\prime} v^{\prime}}}
\end{aligned}
$$

For calculation of effective diffusivities, a turbulent closure model is needed. How physical properties changing with temperature influence the turbulent 
diffusivity expressions has not been systematically investigated [6]. Therefore, many different assumptions have been proposed for models based on mixing length concept, which were originally developed for fluid with constant properties [20]. Semi-empirical correlations exist which express turbulent diffusivities as functions of a dimensionless distance from the wall $y^{+}=(R-r) \sqrt{\bar{\rho}_{z} \tau_{w}} / \mu_{z}$, which can be useful to characterize the fluid-wall interaction [20]. Some authors assumed that the original correlations may be used without changes, if proper values of physical properties are considered to compute dimensionless distance $y^{+}$. Deissler [21] suggested to use the wall properties $\left(\bar{\rho}_{z}=\bar{\rho}_{w}\right.$ and $\left.\mu_{z}=\mu_{w}\right)$, Sastry [22] the local properties $\left(\bar{\rho}_{z}=\bar{\rho}\right.$ and $\left.\mu_{z}=\mu\right)$ and Goldmann [23] proposed an integral formulation between previous extremes, based on a theoretical hypothesis on local turbulence characteristics.

$$
y^{+}=\int_{R-r}^{R} \frac{\sqrt{\bar{\rho} \tau_{w}}}{\mu} d r
$$

The method suggested by Goldmann produces better agreement with experimental heat transfer data [6].

The discussed features are shared by all considered models. Essentially they differ for the way of computing the usual turbulent diffusivities with variable thermophysical properties and the corrective factors due to density fluctuations. Three physical models will be considered: the model of Petrov and Popov [16,10], the model of Bellmore and Reid [24] and finally the suggested model.

Petrov and Popov applied the mixing length model to calculate the turbulent diffusivities. They adopted the Sastry's approach to take into account variable thermophysical properties but considered an additional corrective procedure to compute effective values, based on the discrepancy between shear stresses computed with constant and variable properties. They totally neglected the effects due to density fluctuations and so implicitly assumed $F_{x x}^{\mu}=F_{r r}^{\lambda}=1$. Bellmore and Reid applied the mixing length model too and adopted the Goldmann approach to take into account variable thermophysical properties. They suggested an innovative method to include density fluctuations in the equations of turbulent transport. The main topics of mixing length theory will be summarized in order to understand the approach suggested by Bellmore and Reid. In the following expressions, a new formal convention for time averaging will be temporarily considered $\langle z\rangle \equiv \bar{z}$. The mixing length theory is based on two heuristic assumptions [20].

$$
\begin{aligned}
& \left\langle u^{\prime} v^{\prime}\right\rangle=+C_{u v}\left\langle\left|u^{\prime}\right|\right\rangle\left\langle\left|v^{\prime}\right|\right\rangle \\
& \left\langle h^{\prime} v^{\prime}\right\rangle=-\zeta C_{h v}\left\langle\left|h^{\prime}\right|\right\rangle\left\langle\left|v^{\prime}\right|\right\rangle
\end{aligned}
$$


where $\zeta=\partial_{r} \bar{h} /\left|\partial_{r} \bar{h}\right|, 0<C_{u v}<1$ and $0<C_{h v}<1$. The signs in both expressions are due to experimental evidence. In particular the sign in equation (23) depends on the fact that $v$ is the velocity component along radial direction pointing into the wall. If the average absolute deviations $\langle|x|\rangle$ were substituted by the standard deviations $\left\langle x^{2}\right\rangle^{1 / 2}$ in the heuristic assumptions, the correlation coefficients would appear. If the velocity fluctuations are normally distributed, there is a definite relationship between the average absolute deviation and the standard deviation [25]. Unfortunately no proof exists ensuring that velocity fluctuations are always normally distributed, particularly for channel flow which is characterized by inhomogeneous turbulence [19]. For this reason, robust statistics, i.e. average absolute deviation, is preferable and the expressions $(23,24)$ will be considered as the definitions of robust correlation coefficients. Each average absolute deviation can be expressed by transverse velocity gradient or transverse enthalpy gradient: this means $\left\langle\left|u^{\prime}\right|\right\rangle=l_{u}\left|\partial_{r} \bar{u}\right|$, $\left\langle\left|v^{\prime}\right|\right\rangle=l_{v}\left|\partial_{r} \bar{u}\right|$ and $\left\langle\left|h^{\prime}\right|\right\rangle=l_{h}\left|\partial_{r} \bar{h}\right|$. Introducing these expressions into previous assumptions $(23,24)$ and grouping the unknown terms, two essential quantities emerge: the mixing length $l_{m}=\left(C_{u v} l_{u} l_{v}\right)^{1 / 2}$ and the turbulent Prandlt number $\operatorname{Pr}_{t}=C_{u v} l_{u} /\left(C_{h v} l_{h}\right)$.

$$
\begin{aligned}
\left\langle u^{\prime} v^{\prime}\right\rangle & =l_{m}^{2}\left|\partial_{r} \bar{u}\right|^{2} \\
\left\langle h^{\prime} v^{\prime}\right\rangle & =-\zeta\left(l_{m}^{2} / P r_{t}\right)\left|\partial_{r} \bar{h}\right|\left|\partial_{r} \bar{u}\right|
\end{aligned}
$$

Both quantities are supplied by turbulent closure models, based on mixing length concept. Bellmore and Reid essentially interpreted the definitions of robust correlation coefficients in a factorized form.

$$
\begin{aligned}
& \left\langle u^{\prime} v^{\prime}\right\rangle=\Delta u \Delta v \\
& \left\langle h^{\prime} v^{\prime}\right\rangle=\Delta h \Delta v
\end{aligned}
$$

where $\Delta u=b_{1}\left\langle\left|u^{\prime}\right|\right\rangle, \Delta v=b_{2}\left\langle\left|v^{\prime}\right|\right\rangle$ and $\Delta h=-\zeta b_{3}\left\langle\left|h^{\prime}\right|\right\rangle$. The congruence with the original heuristic assumptions implies that $b_{1} b_{2}=C_{u v}$ and $b_{2} b_{3}=$ $C_{h v}$ but these constrains are not enough to determine unambiguously the constants $b_{i}$. The equation (25) suggests that the velocity fluctuations along both directions produce comparable effects. The condition $\Delta u=\Delta v$ allows to produce an additional constrain $b_{1} l_{u}=b_{2} l_{v}$. In this way, the values of the constants are found: $b_{1}=\left(C_{u v} l_{v} / l_{u}\right)^{1 / 2}, b_{2}=\left(C_{u v} l_{u} / l_{v}\right)^{1 / 2}$ and $b_{3}=$ $C_{h v} C_{u v}^{-1 / 2}\left(l_{v} / l_{u}\right)^{1 / 2}$. The main advantage of the recasted forms $(27,28)$ is that each function $\Delta z$ depends only on the fluctuations of the same variable $z^{\prime}$ and it can be calculated by means of the mixing length theory. In analogy with this factorization, Bellore and Reid suggested the following general decomposition.

$$
\left\langle\left(u^{\prime}\right)^{n_{1}}\left(v^{\prime}\right)^{n_{2}}\left(h^{\prime}\right)^{n_{3}}\right\rangle=(\Delta u)^{n_{1}}(\Delta v)^{n_{2}}(\Delta h)^{n_{3}}
$$


where $n_{i} \in \mathcal{N}^{+}$. For example, considering $n_{1}=n_{3}=1$ and $n_{2}=0$, the expression for turbulent thermal diffusivity along axial direction can be found.

$$
\left\langle h^{\prime} u^{\prime}\right\rangle=\Delta h \Delta u=-\zeta\left(C_{h v} l_{v} / l_{u}\right)\left\langle\left|h^{\prime}\right|\right\rangle\left\langle\left|u^{\prime}\right|\right\rangle=-\zeta C_{h u}\left\langle\left|h^{\prime}\right|\right\rangle\left\langle\left|u^{\prime}\right|\right\rangle
$$

In the same way, we can proceed with all turbulent terms involved into expressions $(20,21)$, which can be calculated by means of the general decomposition (29). The characteristic velocity due to density fluctuations can be expressed too, by taking into account equations $(28,30)$.

$$
\begin{aligned}
& F_{x x}^{\mu}=F_{r r}^{\lambda}=\phi_{B R}=1+\zeta \beta \sigma_{B R}-\beta^{2} \sigma_{B R}^{2} \\
& \bar{u}_{B R}^{*}=\bar{v}_{B R}^{*}=\zeta \beta\left(l_{m}^{2}\left|\partial_{r} \bar{u}\right| / P r_{t}\right)\left|\partial_{r} \bar{h}\right|
\end{aligned}
$$

where $\sigma_{B R}=\left(l_{m} / P r_{t}\right)\left|\partial_{r} \bar{h}\right|$ can be considered an index of intensity for density fluctuations. We can now discuss the effects due to density fluctuations. Since $\beta \sigma_{B R}$ is usually a small quantity also near the critical point, we can suppose $\phi_{B R} \approx 1+\zeta \beta \sigma_{B R}$. This means that during cooling conditions $(\zeta<0)$, the density fluctuations reduce the turbulent diffusivities $\left(\phi_{B R}<1\right)$, while during heating conditions $(\zeta>0)$ they substantially increase turbulent diffusivities $\left(\phi_{B R}>1\right)$. Additional convective terms along axial direction are negligible. The radial velocity field in absence of density fluctuations $\bar{v}_{0}$ can be discussed by equation (19). Let us define a vectorial velocity in absence of density fluctuations $\overline{\mathbf{w}}_{0}=\left(\bar{u}, \bar{v}_{0}\right)$. For mini/micro channels, the density gradient can be reasonably assumed orthogonal to this velocity $\overline{\mathbf{w}}_{0} \cdot \nabla \rho \approx 0$, because $\overline{\mathbf{w}}_{0}$ is approximately oriented along stream lines. In this way, equation (19) easily allows to find that $-\zeta \bar{v}_{0} \geq 0$. On the other hand, the correlation $\zeta \bar{v}^{*} \geq 0$ yields for definition. Taking into account that $\bar{v} \approx-\bar{v}^{*}$, a similar correlation for effective radial velocity is found $-\zeta \bar{v} \geq 0$. Since $|\bar{v}|>>\left|\bar{v}_{0}\right|$, then the density fluctuations increase convective radial terms both during cooling conditions $\left(\bar{v}>>\bar{v}_{0} \geq 0\right)$ and heating conditions $\left(\bar{v}<<\bar{v}_{0} \leq 0\right)$.

Both the previous models are unsatisfying for some reasons. The distinguishing features will be organized into three different categories: the turbulent closure model for common terms due to time averaging, the procedure to take into account variable physical properties and finally the turbulent closure model for additional terms due to density fluctuations. Both previous models use turbulent models based on mixing length concept. Despite some generalizations were proposed, these models have some drawbacks [19]. Firstly, they strongly depend on the geometry of the flow considered to find practical relations for mixing length and so they are not general. Secondly, they prescribe that turbulent diffusivities are zero where there is no velocity gradient, as it happens for the centerline of mini/micro channel. This second feature is contrary to experimental evidence and it could be relevant for considered application. About variable physical properties, the previous models differ for the consid- 
ered operative procedure, but they substantially adopt common correlations by reducing the problem to calculate a modified $y^{+}$. In particular, they do not modify the functional nature of the correlations. Instead variable properties probably modify the local turbulent phenomena, which only a model based on differential equations can describe. Finally, only the model of Bellmore and Reid considers the density fluctuations. This model is based on the possibility to calculate average absolute deviations by means of mixing length concept. This possibility is not accessible by most widespread turbulence models and it would require to know statistical distribution for each variable. So the discussed method is only valid for the simplest turbulent model and it is not directly generalizable. Moreover, the expression (30), which has been deduced according to generalized decomposition (29), is contrary to gradient-diffusion hypothesis (10) because it depends on radial enthalpy gradient instead of axial one.

A different approach is suggested. Before proceeding to describe the constitutive hypothesis, some explanations on the mathematical meaning of general decomposition suggested by Bellmore and Reid (29) are needed. Within framework of mixing length theory, the turbulent closure model can be considered a tool which allows to calculate robust correlation coefficients $C_{u v}=l_{m}^{2} /\left(l_{u} l_{v}\right)$ and $C_{h v}=l_{m}^{2} /\left(P r_{t} l_{v}^{2}\right)$. Analogously, when the equation (30) is considered, the general decomposition reduces to suppose $C_{h u}=C_{h v} l_{v} / l_{u}$. The coefficients $b_{i}$ involved in factorized heuristic assumptions $(27,28)$ can be expressed as functions of second-order mixed robust correlation coefficients $C_{h v}, C_{h u}$ and $C_{u v}$, which are the non-zero lowest-order coefficients. Substituting these expressions into equation (29), a modified expression is found.

$$
\left\langle\left(u^{\prime}\right)^{n_{1}}\left(v^{\prime}\right)^{n_{2}}\left(h^{\prime}\right)^{n_{3}}\right\rangle=(-\zeta)^{n_{3}} C_{h v}^{q_{1}} C_{h u}^{q_{2}} C_{u v}^{q_{3}}\left\langle\left|u^{\prime}\right|\right\rangle^{n_{1}}\left\langle\left|v^{\prime}\right|\right\rangle^{n_{2}}\left\langle\left|h^{\prime}\right|\right\rangle^{n_{3}}
$$

where $q_{i}=\left(1-2 \delta_{i j}\right) n_{j} / 2 \in \mathcal{Q}$. Mathematically the general decomposition (29) is equivalent to suppose that higher-order robust correlation coefficients are proper combinations of lower-order ones. Since by definition $n_{i}=\left(1-\delta_{i j}\right) q_{j}$ and remembering the expressions for lower-order correlation coefficients $(27,28,30)$, the previous equation can be modified.

$$
\left\langle\left(u^{\prime}\right)^{n_{1}}\left(v^{\prime}\right)^{n_{2}}\left(h^{\prime}\right)^{n_{3}}\right\rangle=(-\zeta)^{n_{3}}\left|\left\langle h^{\prime} v^{\prime}\right\rangle\right|^{q_{1}}\left|\left\langle h^{\prime} u^{\prime}\right\rangle\right|^{q_{2}}\left\langle u^{\prime} v^{\prime}\right\rangle^{q_{3}}
$$

This correlation has been rigorously demonstrated within the framework of the theory developed by Bellmore and Reid and so it can be considered equivalent to decomposition (29). The main advantage is that it involves only quantities, which are calculated by all turbulent closure models because they emerge from time averaging of flow equations with constant properties. Essentially the previous relation assumes a general dependency of terms due to density fluctuations from usual terms due to velocity fluctuations. It can be considered as a constitutive hypothesis without any dependence on a particular turbu- 
lent model. In the following the turbulent-viscosity hypothesis (9) and the gradient-diffusion hypothesis (10) will be considered in order to produce a meaningful example without loss of generality. Applying constitutive hypothesis (34) to all turbulent terms involved into expressions $(20,21)$, we find again the same formal expression for corrective factor $\phi$ which influences effective diffusivities (31), but with a different intensity index $\sigma$.

$$
\sigma=\sqrt{\frac{\lambda_{t}^{2}}{\bar{\rho} \mu_{t}} \frac{\left|\partial_{x} T \partial_{r} T\right|}{\left|\partial_{r} \bar{u}\right|}}
$$

In the same way, we can proceed for the characteristic velocity.

$$
\bar{u}^{*}=\zeta \beta\left(\lambda_{t} / \bar{\rho}\right)\left|\partial_{x} T\right|<<\bar{v}^{*}=\zeta \beta\left(\lambda_{t} / \bar{\rho}\right)\left|\partial_{r} T\right|
$$

Since these relations involve temperature gradient, contrarily to previous ones which involve enthalpy gradient, the effects due to compressibility must be discussed. For both axial and radial direction, the generic component of enthalpy gradient can be expressed by means of temperature and pressure changes $\partial_{i} \bar{h}=c_{p} T\left[\partial_{i} T / T-\varphi \partial_{i} p / p\right]$, where $c_{p}$ is the specific heat $c_{p}=\left.\partial_{T} \bar{h}\right|_{p}$ and the dimensionless parameter $\varphi$ takes into account non-ideal gas effects.

$$
\varphi=\frac{\beta c_{p} T-1}{\bar{\rho} c_{p} T / p}
$$

In all the following calculations, this parameter is included into the range $0<\varphi<0.21$. Since the relative temperature changes are much greater than relative pressure changes $\partial_{i} T / T>>\partial_{i} p / p$, then the compressibility effects on enthalpy can be neglected and an approximate relation yields $\partial_{i} \bar{h} \approx c_{p} \partial_{i} T$. According to boundary layer theory, this approximation is even more satisfied along radial direction. For comparing the previous results with those obtained by Bellmore and Reid, the correlations $(31,32)$ will be directly generalized by expressing mixing length and turbulent Prandtl number as functions of turbulent diffusivities. Remembering that $l_{m}=\mu_{t}^{1 / 2}\left(\rho\left|\partial_{r} \bar{u}\right|\right)^{-1 / 2}$ and $\operatorname{Pr}_{t}=$ $\mu_{t}\left|\partial_{r} \bar{h}\right|\left(\lambda_{t}\left|\partial_{r} T\right|\right)^{-1}$, the generalized expressions for the intensity index and for the components of characteristic velocity can be found.

$$
\begin{aligned}
\sigma_{B R} & =\sqrt{\frac{\lambda_{t}^{2}}{\bar{\rho} \mu_{t}} \frac{\left|\partial_{r} T\right|^{2}}{\left|\partial_{r} \bar{u}\right|}} \\
\bar{u}_{B R}^{*} & =\bar{v}_{B R}^{*}=\zeta \beta\left(\lambda_{t} / \bar{\rho}\right)\left|\partial_{r} T\right|
\end{aligned}
$$

Despite the simplicity of deduction procedure, the expressions $(38,39)$ can be calculated by any turbulent model too. Unfortunately they substantially 
describe in the same way the effects due density fluctuations along axial and radial direction. If density fluctuations are due to enthalpy fluctuations and the latter ones satisfy the gradient-diffusion hypothesis (10), which is strongly anisotropic, it is not clear why the effects due density fluctuations should be isotropic. Since the original formulation of Bellmore and Reid was developed for boundary layer flow, the generalized expressions $(38,39)$ overestimate the effect of axial density fluctuations and they can not be universally valid. Here the essential feature of suggested model emerges. The correlations $(35,36)$ involve the axial gradient to predict the effects due to density fluctuations along axial direction. This feature essentially predicts a lower effect of density fluctuations on turbulent diffusivities since $\sigma<<\sigma_{B R}$, because usually $\left|\partial_{x} T\right|<<\left|\partial_{r} T\right|$. About effects on convective terms, the two formulations are formally equivalent for radial direction $\bar{v}^{*}=\bar{v}_{B R}^{*}$, while they again differ with regards of axial direction $\bar{u}^{*}<<\bar{u}_{B R}^{*}$. Since the latter effect is negligible in considered application, the essential difference between the two approaches for simulation of mini/micro channels lies in the description of effective diffusivities and, in particular, in the fact that $|\phi-1|<<\left|\phi_{B R}-1\right|$.

Any closure turbulent model can applied to calculate turbulent diffusivities into equations $(35,36)$. As discussed previously, it is recommended to adopt models based on differential equations. They can easily take into account effects due to variable physical properties because they need no particular correlations, as those involved in mixing length model developed for constantproperty flows [19]. Additional differential equations increase the computational effort but there is no need to include additional terms due to fluctuating properties within these equations, since they are directly formulated for timeaveraged quantities. In the following a couple of two-equation models will be considered in order to compare the effects due to the description of turbulent diffusivities. The standard $k-\epsilon$ model [26] is considered because of its popularity in many engineering fields. It is based on the solution of two separate transport equations which allow to compute turbulence kinetic energy $(k)$ and its dissipation rate $(\epsilon)$ in order to estimate turbulent diffusivities [27]. The other considered model is the RNG $k-\epsilon$ model [28] which can be rigorously developed by means of renormalization group theory. Briefly RNG $k-\epsilon$ model significantly improves the accuracy for near-wall flows, by considering more accurate transport equations and a variable ratio between turbulent viscosity and turbulent thermal conductivity, contrarily to previous model. Original formulation included the possibility to apply two-equation approach very close to the wall too, i.e. at low Reynolds numbers. In the discussed simulations, this possibility will not be used. In this way, the two models are based on the same number of equations in all calculation sub-domains. Since both the two-equation models were formulated for fully-developed turbulence, they are not usually applied in the near-wall region [27]. In the region where the effect of molecular viscosity can not be neglected (approximately $0<y^{+}<60$ ), an additional resolution technique must be supplied. Usually a smaller number of equations is used in this region. Semi-empirical algebraic correlations, 
which involve no differential equation, were developed for flows with constant properties and they are conventionally defined as wall functions. As previously discussed for mixing length correlations, variable physical properties compromise the suitability of wall functions in considered application. The minimum successful strategy, i.e. one-equation model, will be adopted in the near-wall region. The whole calculation domain is subdivided into a viscosity-affected region, which is a little wider than laminar viscous sublayer in order to include the transition layer, and a fully-turbulent region [Pope]. In the latter region, the standard $k-\epsilon$ and the RNG $k-\epsilon$ models can be applied, while in the viscosity-affected region the turbulent diffusivities are assumed as exclusive functions of turbulence kinetic energy [29,30]. A proper blending function [31] between previous calculation procedures completes the method, which is usually referred as two layer zonal model.

A proper set of boundary conditions are need to solve the system of equations. At the inlet boundary, some unknown quantities, which describe the fluid flow, are supposed uniformly distributed along radial direction: $\bar{u}(0, r)=u_{0}$, $\bar{v}(0, r)=0$ and $T(0, r)=T_{0}$. In same way, we can proceed for turbulent quantities involved into two-equation models, i.e. the turbulence kinetic energy $k(0, r)=k_{0}$ and the turbulence dissipation rate $\epsilon(0, r)=\epsilon_{0}$. Usual relations are used to calculate turbulence quantities by means of more convenient quantities involved into fluid flow, such as the average Reynolds number and the mean axial velocity [27]. At the outlet boundary, the only calculation unknow which was not considered within inlet conditions, i.e. the pressure, is imposed $p(L)=p_{L}$. Since the solution is necessarily axisymmetric, no radial gradient is allowed for any solved quantity at the centerline of the mini/micro channel. At the wall boundary a given thermal flux $\partial_{r} T=q_{w} / \lambda$ or, alternatively, a given wall temperature $T(x, R)=T_{w}$ is considered. Velocity components are specified according to no-slip boundary condition: $\bar{u}(x, R)=0$ and $\bar{v}(x, R)=0$. Since near the wall laminar boundary layer exists, similar conditions yield for turbulent quantities: $k(x, R)=0$ and $\epsilon(x, R)=0$. The previous set of physical conditions is insufficient to determine a well-posed mathematical problem: additional informations are produced by linear extrapolation of interior computational domain. This strategy allows a more stable resolution process than other one, based on splitting between physical quantities at the inlet and extrapolated quantities at the outlet. Since the pressure gradients along short mini/micro channels are negligible, this strategy will not reduce the physical accuracy.

For fluid at supercritical pressure near the critical point, the precise measurement of physical properties is not easy. Technical advances probably justify some discrepancies among physical property databases, developed during the last years. These inaccuracies could obviously affect the numerical simulations. In particular the correlation of Petrov and Popov was derived by interpolation of some numerical tests, which adopted a merging database based on two different sets of experimental data [10]. They tried to overcome the lack of description for thermal conductivity near the pseudo-critical temperature, 
which characterized the experimental results of Altunin [32]. In the following calculations, a recently developed database for thermophysical properties of carbon dioxide is considered [database]. For this database, the estimated uncertainty ranges are $0.15 \div 1.5 \%$ in specific heat capacity, less than $5 \%$ in thermal conductivity, $0.03 \div 0.05 \%$ in density and finally $0.3 \div 5 \%$ in viscosity. In the previous ranges, the highest values refer to liquid-like states or the highest pressures.

\section{$3 \quad$ Numerical discretization and solution procedure}

The governing equations for continuity (13), momentum (14) and energy (15) are discretized, according to finite volume method [34]. Essentially the solution domain is subdivided into a finite number of small control volumes. In order to avoid unrealistic solutions, there is a significant benefit to be obtained by arranging unknowns for velocity components on different grid from the one used for all other variables. This strategy, called staggered grid, is adopted [35]. This means that unknown velocity components are located at the faces of control volumes, which surround the computational nodes for residual variables. Since there are two different computational grids, some interpolations are needed to complete lacking information. In particular to solve the momentum equation (14), the face-centered values for pressure are interpolated using momentum equation coefficients and this allows to estimate the effective viscosity at the volume-centered node [27]. Some interpolations are need for convective terms too. In this case, the convective term in the momentum equation is non-linear and it seems to complicate the resolution process. Since the final solution process will be essentially iterative, the non-linear terms can be approximated at each iteration. The outer iteration can be used to estimate the non-linear coefficients. This essentially linearizes the momentum equation and makes it similar to the other ones [34]. The general upwind scheme is adopted to calculate the convective terms for all linearized equations [35]. It sets the face-centered values for all variables equal to the volume-centered values in the upstream volumes. The identification of upstream volumes is done according to approximated velocity field. The previously discussed turbulent models, which take into account the effects due to density fluctuations, involve in the governing equations additional source terms. How to discretize these source terms is reported into Appendix A.

Before proceeding with resolution process, how to choose discretization strategy is discussed. For the considered application, both the thermophysical database and the computational domain must be properly discretized. Since for short mini/micro channels the pressure drops are negligible, the thermophysical properties can be considered as functions only of temperature. Physical properties can be grouped into two different sets. The first set, which 
includes the specific heat $c_{p}$, the thermal conductivity $\lambda$ and the modified compressibility $\beta$, involves thermophysical properties which show a peak near the pseudo-critical temperature for a given range of supercritical pressures. For this reason they are defined non-monotonic properties. The second set, which includes density $\rho$ and dynamic viscosity $\mu$, involves thermophysical properties which decrease monotonically with temperature. The considered database [33] discriminates property changes due to very small temperature differences, which near the critical temperature are approximately comparable to $1 \mathrm{mK}$. The high-resolution capacity of this database produces a great amount of information, which would slow down the upgrade of physical properties during resolution process. For this reason, piecewise linear approximations will be adopted and the distribution of nodal values will be properly chosen. If the generic property $f(T)$ is considered, the problem reduces to find the optimal distribution $T_{i}$ where $1 \leq i \leq N_{f}$ and the corresponding one $f_{i}=f\left(T_{i}\right)$ which ensure desired accuracy. Between two consecutive nodal values $T_{i}$ and $T_{i+1}$, the function will be approximated by means of a linear function $\tilde{f}_{i, i+1}(T)=f_{i}-\left(f_{i+1}-f_{i}\right)\left(T-T_{i}\right) /\left(T_{i+1}-T_{i}\right)$ where $T_{i}<T<T_{i+1}$. A local relative error $\tilde{e}_{i, i+1}=\max \left(\left|f-\tilde{f}_{i, i+1}\right| / f\right)$ can be assigned to the same temperature range and a global relative error $e=\max _{i}\left(\tilde{e}_{i, i+1}\right)$ can be assigned to the whole temperature range by considering every $i$ into range $1 \leq i \leq N_{f}-1$. If the initial temperature $T_{1}$ is given, the optimal distribution can be unambiguously defined as the minimum number of nodal values which guaranties that global relative error is upper-bounded $e_{f} \leq e_{f}^{0}$. The parameter $e_{f}^{0}$ is the error threshold for considered piecewise linear approximation of generic property $f(T)$. This parameter determines the smallest temperature difference involved into whole approximation $\Delta T_{m}\left(e_{f}^{0}\right)=\min _{i}\left(T_{i+1}-T_{i}\right)$ for every $i$ into range $1 \leq i \leq N_{f}-1$. For definition $\Delta T_{m}(0)$ represents the resolution of original database. Each piecewise approximation allows to reduce the number of nodal values by increasing the smallest temperature difference $\Delta T_{m}\left(e_{f}^{0}\right) \geq \Delta T_{m}(0)$. Now this approach will be turned over. The total number $N_{f}$ of nodal values will be considered a fixed constrain for all the physical properties and for all supercritical pressures. This constrain depends on what run time can be considered acceptable in considered application. In this way, the highest error thresholds will appear for non-monotonic properties and for the lowest supercritical pressure, i.e. where the strongest changes exist. In the following calculations, the error thresholds are $0.3 \div 5.6 \%$ for heat capacity, $0.1 \div 2 \%$ for thermal conductivity, $0.1 \div 0.5 \%$ for modified compressibility and $0.1 \div 0.5 \%$ for both monotonic properties. For density and specific heat, the piecewise linear approximations produce errors greater than estimated uncertainties for the same quantities. In particular error threshold for heat capacity appears quite high. The minimum temperature difference involved into this approximation is sensitively greater than resolution of original database $\Delta T_{m}(0.056)=5 m K>1 m K$, but it is smaller than temperature differences which the discretized computational domain allows to estimate.

According to boundary layer theory, the unknown quantities are characterized 
by axial gradients which are much smaller than radial ones. For this reason, an axially homogeneous mesh and a characteristic length of generic control volume $\Delta x$ comparable to radial dimension can be adopted. Three different meshes were considered in order to test the dependence of the results on this parameter and they produced approximately the same results. For this reason, the range $\Delta x / R \approx 0.13 \div 1.4$ is suggested.

The radial direction needs additional care. As previously discussed, variable thermophysical properties induce to prefer one-equation model in comparison with wall functions for near-wall treatment. The discretized domain should be very fine near the wall in order to solve this equation into laminar viscous sublayer $\left(y^{+}<5\right)$. Usually the thickness of the control volume adjacent to the wall $\Delta r_{w}$ is determinated such that the dimensionless distance $y_{w}^{+}$of the centroid is approximately unitary [36]. In this case, no ambiguity exists for calculation of physical properties since the considered point is very close to the wall. This practice allows to estimate thickness of the control volume adjacent to the wall.

$$
\Delta r_{w} \approx \Delta r_{w}^{+}=\min _{x}\left(\frac{2}{\sqrt{\bar{\rho}_{w} \tau_{w} / \mu_{w}^{2}}}\right)
$$

Radially homogeneous mesh would increase enormously the computational time. For this reason, the thickness of the control volume adjacent to the axis of the mini/micro channel $\Delta r_{a}$ is assumed much greater than previous one $\Delta r_{a}>\Delta r_{w}$. A geometric progression with ratio $\chi=\left(R-\Delta r_{a}\right) /\left(R-\Delta r_{w}\right)$ can be assumed to properly blend the previous extremes. The total number of elements $N_{a, w}$ depends only on extreme thicknesses.

$$
N_{a, w}=1+\Phi\left\{\frac{\ln \left(\Delta r_{w} / \Delta r_{a}\right)}{\ln \left[\left(R-\Delta r_{a}\right) /\left(R-\Delta r_{w}\right)\right]}\right\}
$$

where $\Phi: \mathcal{R} \rightarrow \mathcal{N}$ rounds the argument to the nearest natural number towards infinity.

For turbulent convective heat transfer at supercritical pressure near the critical point, the condition $y_{w}^{+} \approx 1$ is not the only one and it could not be the more severe. Since the easiest way to approximate the solution between two consecutive nodal values is to consider a linear function [35], an error could occur in estimating the physical properties if too coarse meshes are considered. If very high-density meshes are avoided [7], only a local grid refinement can completely solve this problem [34]. In the following an easy strategy is suggested for this particular application in order to find quickly the proper mesh. The basic idea is to guarantee that temperature difference between two adjacent control volumes is small enough to produce acceptable errors in estimating maximum specific heat. In the worst case, the peak of specific 
heat is included midway between two consecutive nodal values. The minimum temperature difference $\Delta T_{c}$ is selected such as to estimate the maximum value of specific heat within the limits due to error threshold considered in the discretization of thermophysical database. This local constrain involves only the maximum value of specific heat, depends on the peak width and it may differ from $\Delta T_{m}$ which derives from a global constrain. For the lowest supercritical pressure considered in this study $(7.412 \mathrm{MPa})$, an acceptable value is $\Delta T_{c} \approx 2 \Delta T_{m}(0.056)=10 \mathrm{mK}$. If the considered calculation involves the pseudo-critical temperature, i.e. $T_{0} \geq T_{p c} \geq T_{w}$ for cooling or $T_{0} \leq T_{p c} \leq T_{w}$ for heating, the equation $T(x, r)=T_{p c}$ implicitly defines the coordinates of pseudo-critical temperature all over the computational domain. If the pseudo-critical temperature is involved into a given transverse section of mini/micro channel, then the radial coordinate, which identifies this temperature, is unique. The previous relation can be made explicit by considering $r=\eta(x)$. According to assumed boundary conditions, the initial value is known $\eta(0)=R$. Since this function is monotonically decreasing $d_{x} \eta \leq 0$, the inverse function exists $x=\eta^{-1}(r)$. Taking advantage of this result, the local constrain can be expressed for every radial coordinate which identifies the pseudo-critical temperature within computational domain.

$$
\Delta r<\Delta r_{c}=\frac{\Delta T_{c}}{\left|\partial_{r} T\left(\eta^{-1}, r\right)\right|}
$$

Contrarily to criterion (40), the previous one depends on solution because the position $\eta^{-1}(r)$ of pseudo-critical temperature is not given at the beginning. Two main cases can be distinguished. If the axial change within computational domain is small $\left|d_{x} \eta\right|<<R / L$, then the final value does not differ consistently from initial value $\eta(L) \approx R$. This condition is realized when small thermal fluxes at the wall or wall temperatures close to pseudo-critical temperature are considered. In this case, the condition (42) can be applied for a very thin buffer region $\delta_{w}>R-\eta(L)$ where the grid can be homogeneously constructed finer than remaining domain. The radial discretization step can be selected by considering the more severe between the previous constrains $(40,42)$. The remaining domain can be discretized by means of usual geometric progression. The generic radial temperature gradient $\left|\partial_{r} T(x, r)\right|$ involving the pseudo-critical temperature is reported into Fig. 1. Contrarily to constant property fluids, the temperature gradient is non-monotonic and a local minimum occurs for pseudo-critical temperature at $r=\eta(x)$. In this example, which corresponds to the first validation test discussed in the following section, the pseudo-critical temperature is well confined within the buffer region $\delta_{w}=20 \Delta r_{w}$. It is interesting to note that $\left|\partial_{r} T\left(\eta^{-1}, r\right)\right|$ decreases moving away from the wall. For this reason, the critical discretization step $\Delta r_{c}$ increases moving away from the wall. It will be more difficult to satisfy the condition (42) at the inlet of mini/micro channel because in this region the 


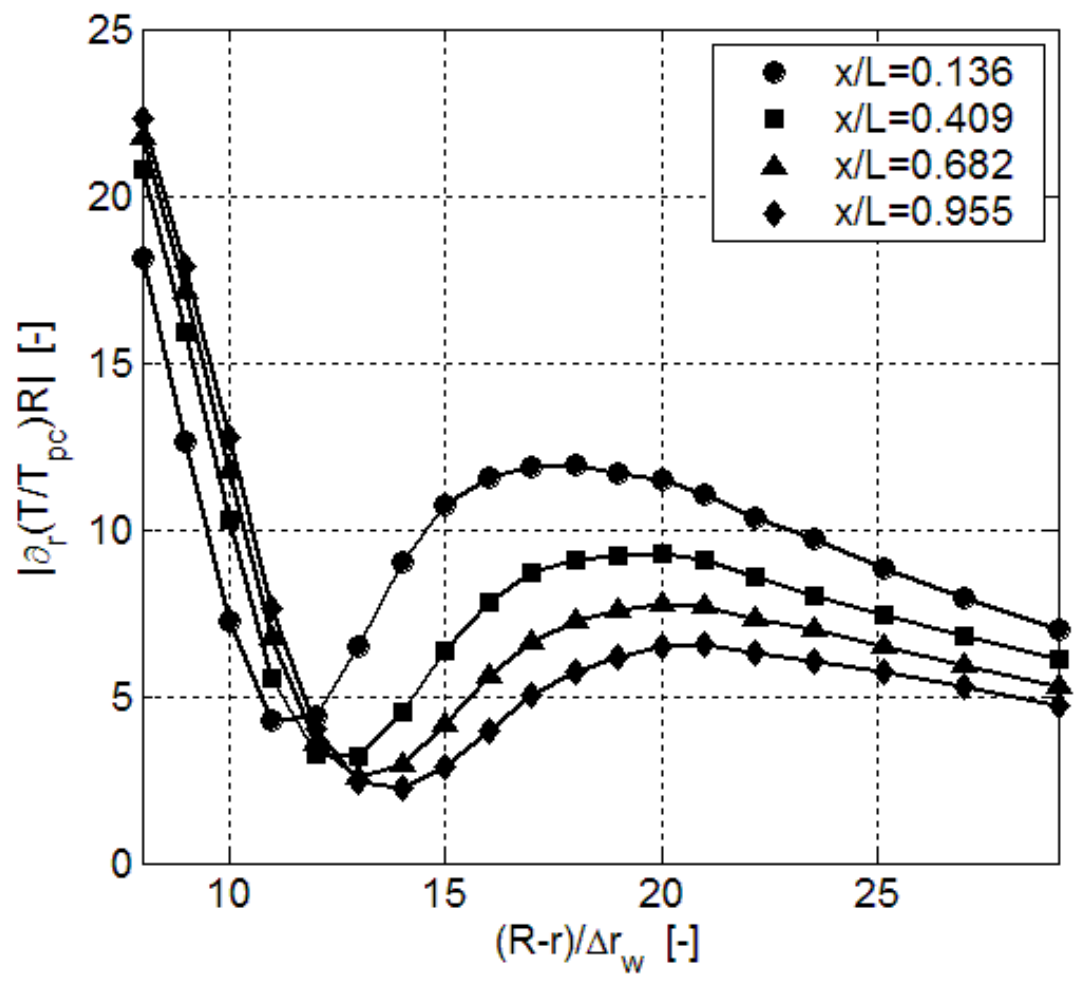

Fig. 1. Effect of pseudo-critical temperature on radial temperature gradients. Different axial locations are considered $x / L=0.136 \div 0.955$. Adopted mesh is homogeneously defined for $(R-r) / \Delta r_{w}<20$ according to critical condition $\Delta r_{w} / R=\Delta r_{c}^{m} / R=1 \times 10^{-5}$ and then made coarser by geometric progression.

pseudo-critical temperature is located near the wall. Practically this problem can be overcome by considering a modest forcing of previous condition. Assuming that the condition (42) is satisfied only for $x>x_{c}$ where $x_{c}<<L$, i.e. only for $r<r_{c}$ where $R-r_{c}<<\delta_{w}$, the limiting threshold for radial discretization into buffer region due to pseudo-critical temperature can be found $\Delta r_{c}^{m}=\Delta T_{c} /\left|\partial_{r} T\left(\eta_{c}^{-1}, r_{c}\right)\right|$. Considering $x_{c} / L=0.136$ in the previous example, the limiting radial discretization in the buffer region due to pseudo-critical temperature is much more severe than usual one (40) due to low-Reynolds turbulence models $\Delta r_{c}^{m} / \Delta r_{w}^{+} \approx 0.129$.

If the radial position of pseudo-critical temperature changes substantially within computational domain, then the final value differs from initial value $\eta(L)<R$ or eventually, for an axial coordinate $x_{a}$, the pseudo-critical temperature intersects the centerline $\eta\left(x_{a}\right)=0$. It is not possible to define in this case a buffer region where the pseudo-critical temperature is confined. However in this case the weak strategy of satisfying condition (42) only for $x>x_{c}$ where $x_{c}<<L$ implies that the limiting threshold for radial discretization is located farer from the wall because $\eta_{c} / x_{c} \propto\left|d_{x} \eta\right|>>R / L$. If the radial thickness required by the most severe threshold were the same previously considered, this moving away from the wall would simply increase the high- 


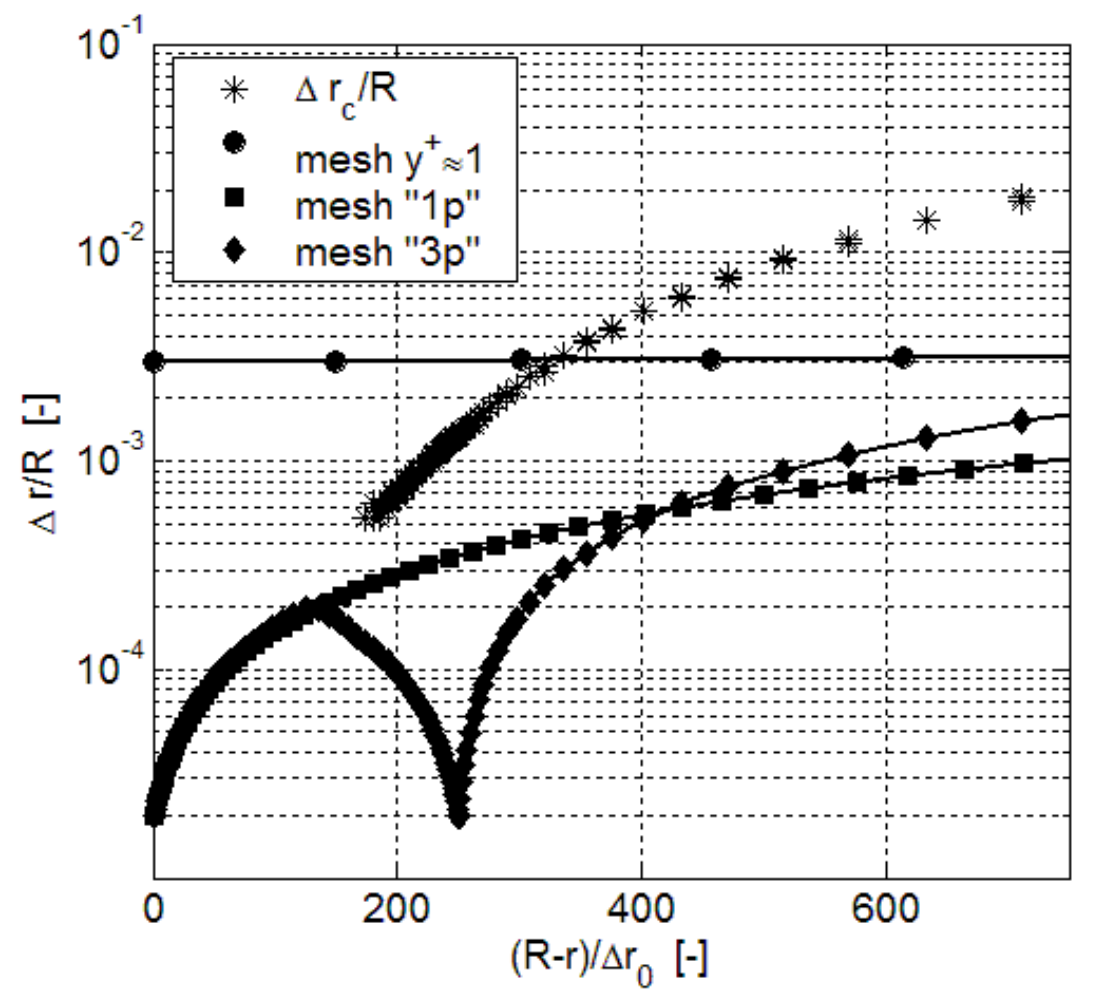

Fig. 2. Critical discretization steps due to pseudo-critical temperature. The limiting threshold is $\Delta r_{c}^{m} / R=5 \times 10^{-4}$ at $x_{c} / L=0.018$. Different radial meshes with the same number of nodes are also reported. Labels "1p" and "3p" refer to the number of constitutive geometric progressions. The normalization value $\Delta r_{0} / R=2 \times 10^{-5}$ is assumed equal to the minimum among first-wall thicknesses for considered meshes.

resolution portion of the mesh. Instead $\left|\partial_{r} T\left(\eta^{-1}, r\right)\right|$ decreases moving away from the wall and for this reason the limiting threshold $\Delta r_{c}^{m}$ allows in this case coarser meshes. In Fig. 2 a comparison among different meshes, which refers to the experimental runs discussed in the following section, is reported together with critical discretization steps due to pseudo-critical temperature. Despite the fact that $x_{c}$ is much smaller than the value previously considered in the first validation test $x_{c} / L=0.018<<0.136$, the limiting radial threshold is weaker $\Delta r_{c}^{m} / R=5 \times 10^{-4}>>1 \times 10^{-5}$. Unfortunately in this case the condition (42) must be satisfied for the whole computational domain and also when the radial discretization proceeds towards the centerline. The first idea is to construct a mesh characterized by an higher resolution at the wall and then verifies that the geometric progression satisfies the condition (42) for any radial coordinate. This strategy corresponds to the single-progression mesh reported in Fig. 2. If preliminary results allow to estimate the limiting condition due to pseudo-critical temperature, multiple-progression meshes can be considered to optimally distribute nodal values along radial direction. Also in this case, the limiting radial discretization due to pseudo-critical temperature is much more severe than usual one (40) due to low-Reynolds turbulence 
models $\Delta r_{c}^{m} / \Delta r_{w}^{+} \approx 0.167$.

If a first estimation of the solution is given, then the previous considerations allow to choose a refined mesh, which properly satisfies the constrains due to pseudo-critical temperature. Since in many cases these constrains are the most severe which affect the solution process, usually the refined mesh ensures the mesh-independence of the solutions. Therefore this strategy reduces to consider a couple of meshes to solve the problem: a first guess mesh to estimate the solution and a second refined mesh, which depends on how the radial position of pseudo-critical temperature changes in the computational domain, i.e. if $\left|d_{x} \eta\right|<<R / L$ or $\left|d_{x} \eta\right|>>R / L$. Let us consider again the first validation test, which will be presented moreover. Since in this case the wall thermal flux is fixed, the wall temperature distribution can be used to check if the solution is mesh-independent. Since all meshes share the same axial discretization, each wall temperature distribution can be compared with the final solution by reporting the averaged value and the standard deviation of wall temperature discrepancies. A first guess mesh with 60 radial nodes and $y_{w}^{+} \approx 1$ produces wall temperature discrepancies equal to $121.0 \pm 90.0 \mathrm{mK}$, which are much higher than $\Delta T_{c}=10 \mathrm{mK}$. The problem is not overcome by simply refining mesh at the wall: a second guess mesh with 80 radial nodes and $y_{w}^{+} \approx 0.1$ produces wall temperature discrepancies equal to $55.0 \pm 79.0 \mathrm{mK}$. As previously reported for this case, if the same radial nodes are organized so as to realize a buffer region which always includes the pseudo-critical temperature, the mesh performance is improved and the wall temperature discrepancies reduce to $0.5 \pm 0.4 \mathrm{mK}$. The final solution considered in the previous comparisons was obtained by considering 20 additional radial nodes, which substantially do not improve further the accuracy. The suggested strategy allows to restrict the number of grid nodes to the minimum needed to describe properly the effects of pseudo-critical temperature. The greatest mesh used in the numerical simulations reported moreover is characterized by 118 radial nodes, which are much less than those prescribed by single-progression high-density meshes [7]. Since the discretization strategy is defined, the whole resolution process can proceed. The discretized governing equations for continuity, momentum and energy are solved sequentially in order to realize a solution loop. The SIMPLE algorithm [27] is adopted. It prescribes a relationship between velocity and pressure values which enforces mass conservation and allows to obtain the pressure field. Because these equations are non-linear and highly-coupled by the dependence of thermophysical properties on temperature, several iterations of the solution loop must be performed before a converged solution is obtained. To take into account the effect of density fluctuations on turbulent diffusivities, they are corrected at the end of each iteration by means of parameter $\phi$, calculated according to turbulent models previously discussed. When all the unknown variables are updated by corrective quantities which are small enough to satisfy a given convergence criterion, the solution loop terminates. The convergence criterion is equal to $1 \times 10^{-3}$ for the validation cases [36], which are characterized by fixed wall thermal flux, and it is equal to $1 \times 10^{-5}$ 
for the experimental runs, which are characterized by fixed wall temperature. For each discretized equation, a Gauss-Seidel linear equation solver is used in conjunction with an algebraic multi-grid method to solve the resultant scalar system of equations for the solving variables [27]. In order to prevent divergence, the velocity components in the momentum equations, the temperature in the energy equation and the transport properties are updated by corrective quantities smaller than those due to pure calculation. The reduction factor is called under-relaxation factor. Near the critical point, Lee and Howell [36] suggest to renew iteratively with an under-relaxation factor the thermophysical properties too. The under-relaxation of the thermophysical properties causes the velocity and the temperature fields to respond rather slowly during solution process. This practice realizes a multi-level under-relaxation which allows to control strong instabilities emerging when too coarse meshes are adopted to describe fluid flow near the critical point. If the mesh is chosen according to suggested strategy, there is no need of multi-level under-relaxation and the usual practice can be adopted.

\section{Results and discussion}

\subsection{Comparison with other predictions and experimental data for local heat transfer coefficient}

The present paper aims to investigate the turbulent convective heat transfer in mini/micro channels for carbon dioxide at supercritical pressure in order to numerically verify the existence of heat transfer impairment. Before proceeding in this direction, a comparison with other numerical predictions and experimental data is needed. This comparison allows firstly to verify the reliability of numerical results and secondly to test if the suggested approach for density fluctuations is more efficient than usual approach. The experiments oriented to characterize the convective heat transfer usually aim to measure the local heat transfer coefficient and/or the average heat transfer coefficient. Two common practices are considered. In the first case, the wall thermal flux is imposed and the measurement of the local heat transfer coefficient reduces to the measurement of the wall temperature. In the second case, the wall temperature is imposed by means of a thermostatic device and the measurement of the average heat transfer coefficient is obtained from the heat balance between the wall heat flux and the bulk enthalpy increase of the fluid. In both cases, the bulk enthalpy increase of the fluid is supposed much larger than changes of kinetic and potential energy or the axial heat diffusion at each end of the duct. If the local heat transfer coefficients are known, then the average heat transfer coefficient can be calculated by line integration along the axis but not vice versa. For this reason, the comparison with experimental mea- 


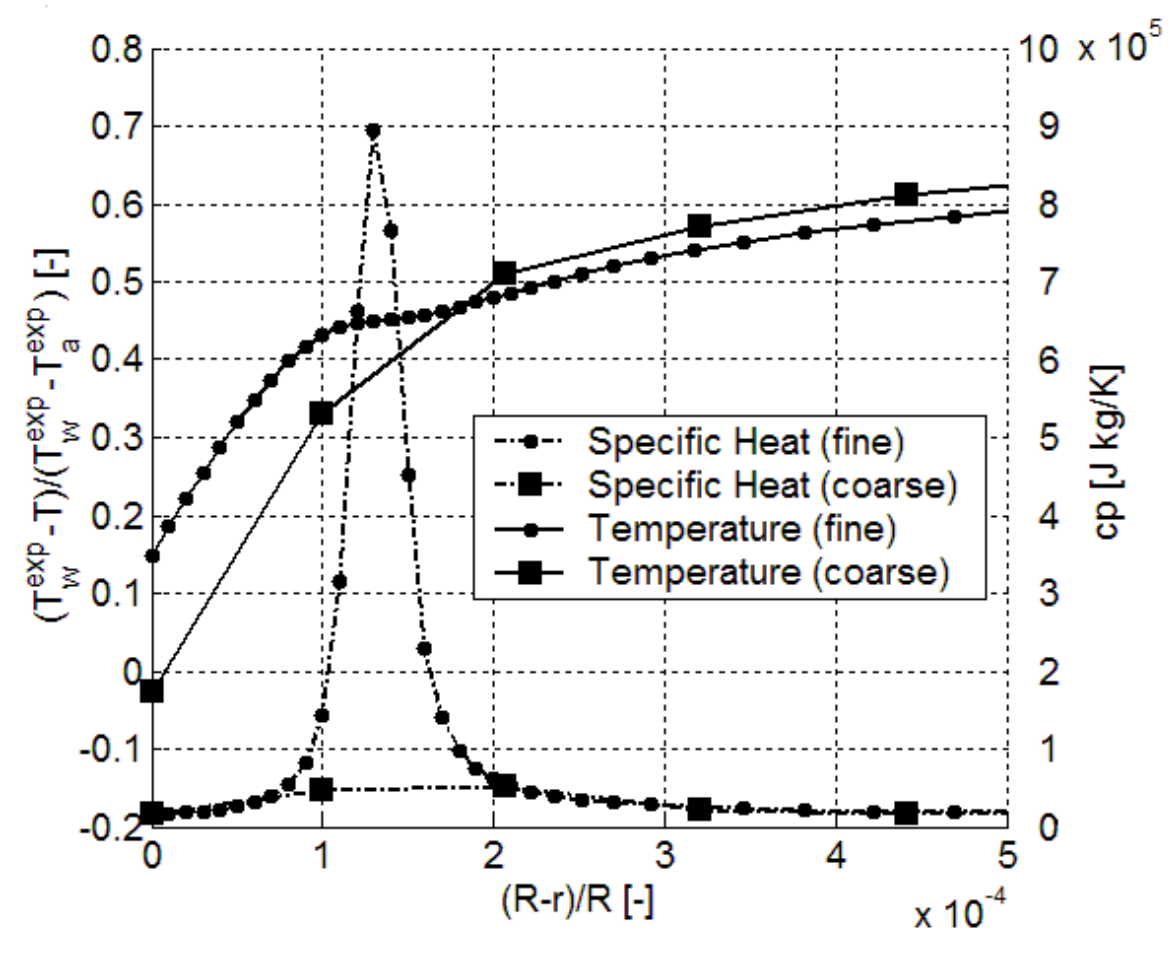

Fig. 3. Effect of radial discretization on the solution. The predicted profiles for specific heat capacity and fluid temperature are both reported in a thin layer near the wall. Both meshes are based on geometric progression. The coarse mesh, which realizes $y_{w}^{+} \approx 1$, is not suitable to describe the peak in specific heat. The reported markers are representative of grid nodes.

surements of local heat transfer coefficients is more meaningful to verify the reliability of the numerical results. Moreover since the numerical results will be used to discriminate among some phenomenological correlations oriented to average heat transfer coefficients, an independent validation step, which involves local heat transfer coefficients, is needed. Due to experimental difficulties, there have been few measurements inside a tube of radial temperature profiles which involve the pseudo-critical temperature. This consideration is even more true for mini/micro channels where the characteristic sizes are prohibitive $(d<2 \mathrm{~mm})$. For this reason, the experimental data for a common duct due to Wood and Smith [37] will be considered. They considered an upward flow of carbon dioxide under heating conditions in a tube with common diameter $(d=22.91 \mathrm{~mm})$ and measured radial temperature profiles by keeping fixed the wall thermal flux. The same set of data was considered for validation purposes by Lee and Howell [36]. Only in this case, the effect of gravity has been added to momentum equation, since condition (1) does not hold.

In Fig. 3 the effect of radial discretization on the solution is reported. Once again the coarse mesh based on condition (40) shows to be not suitable for describing the peak of specific heat capacity. Since the concavity of temperature profile changes because of the peak in specific heat, the coarse mesh introduces 


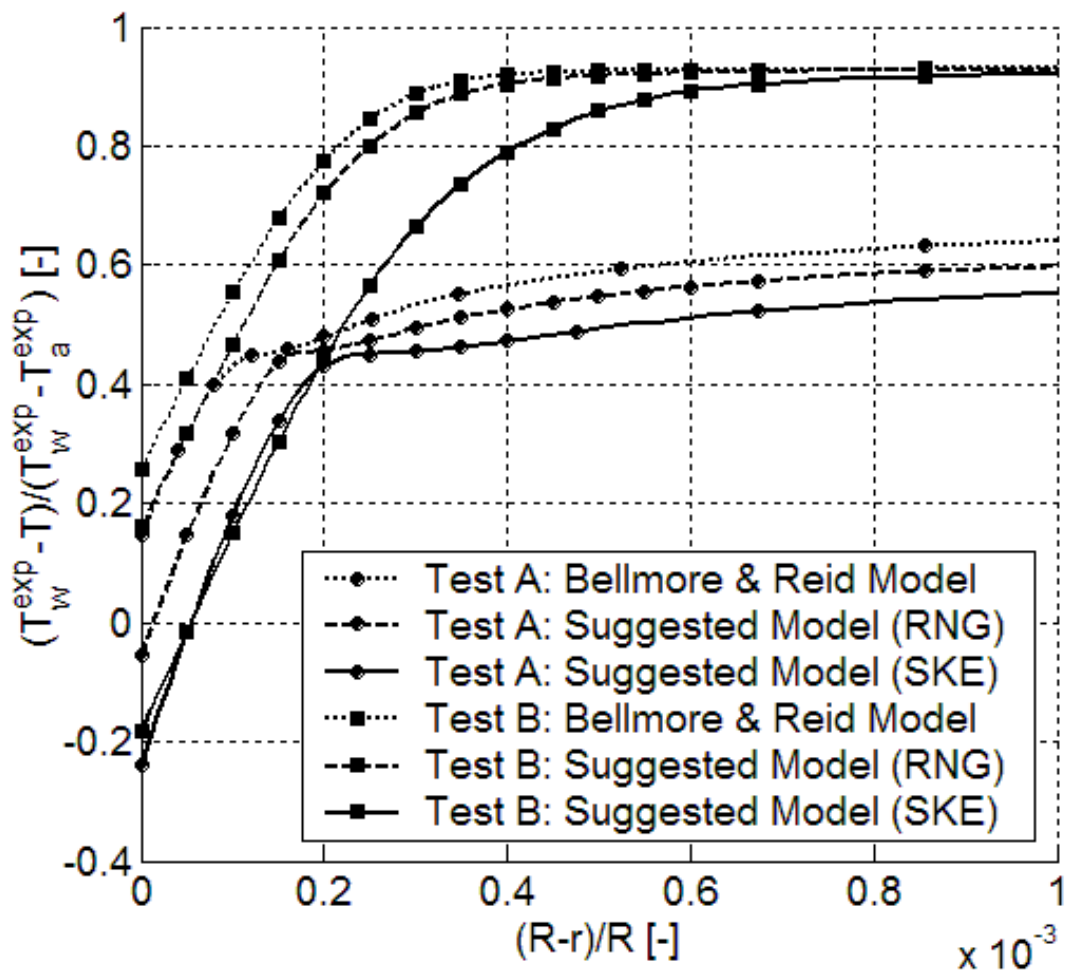

Fig. 4. Comparison of predicted profiles of dimensionless temperature calculated by means of different models with experimental data of Wood and Smith [37] in a thin layer near the wall. The reported cases are different because of the wall thermal flux, which is $63.05 \mathrm{~kW} / \mathrm{m}^{2}$ for Test $A$ and $204.91 \mathrm{~kW} / \mathrm{m}^{2}$ for Test B. The label "RNG" means RNG $k-\epsilon$ model and the label "SKE" means standard $k-\epsilon$ model. The reported markers are not representative of grid nodes.

some errors in predicting the temperature profile and consequently the local heat transfer coefficient. In this particular case, the coarse mesh induces to think that the model of Bellmore and Reid works better than it really does. In this example, the radial discretization induces some errors in the wall temperature which are of the same order of magnitude of uncertainties involved into experimental measurements. The coarse mesh shows a strong unstable behavior because the solution process tries to cut off the peak in specific heat, which behaves like a local numerical noise breaking the smooth solution. This probably justifies the need of multi-level under-relaxation in the numerical simulations performed by Lee and Howell [Howell]. In the following only fine meshes will be considered.

A comparison of predicted profiles of dimensionless temperature calculated by means of different models with experimental data of Wood and Smith [37] in a thin layer near the wall is shown in Fig. 4. The reported cases are different because of the wall thermal flux, which is $63.05 \mathrm{~kW} / \mathrm{m}^{2}$ for Test $A$ and $204.91 \mathrm{~kW} / \mathrm{m}^{2}$ for Test $B$. Because of the high mass flow rate $\left(R e=9.3 \times 10^{5}\right)$, the pseudo-critical temperature is positioned near the wall and it is well con- 


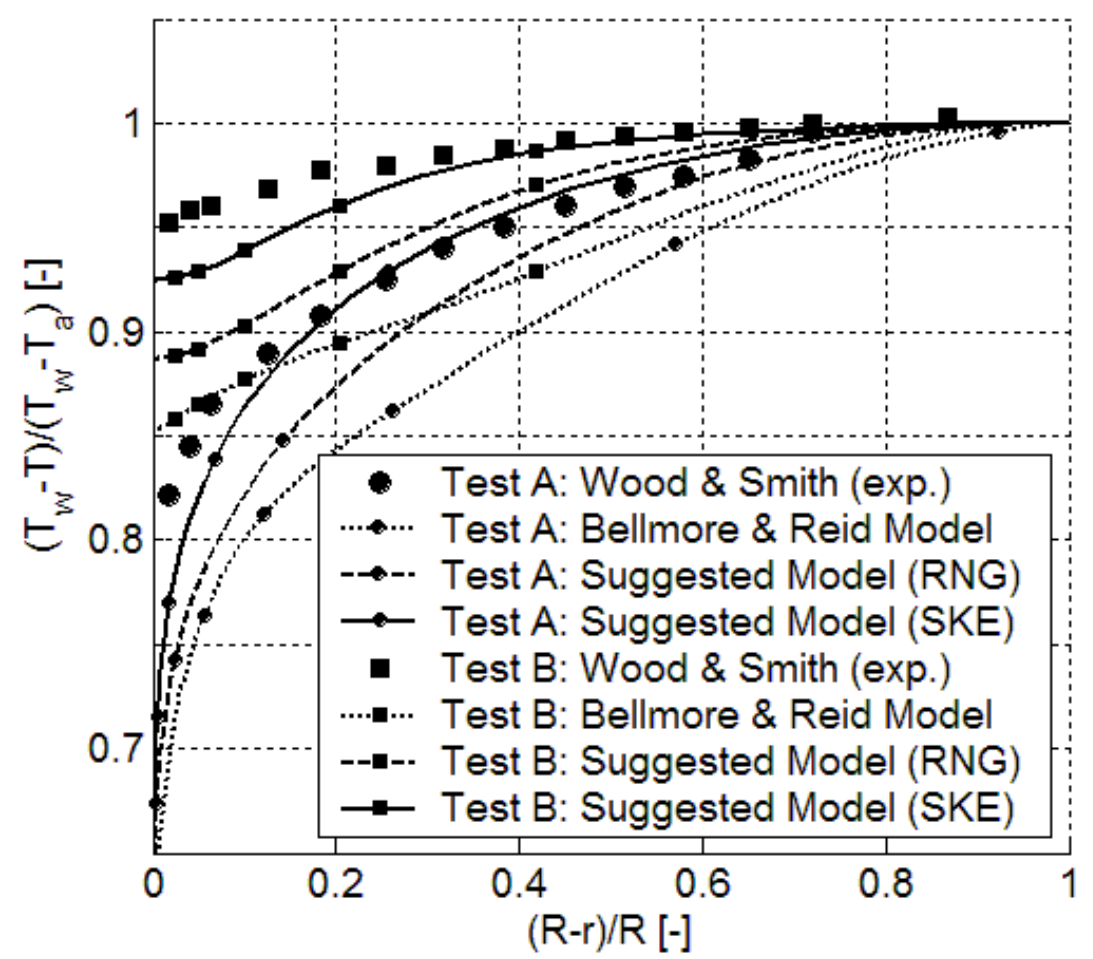

Fig. 5. Comparison of predicted profiles of dimensionless temperature calculated by means of different models with experimental data of Wood and Smith [37] at a given axial location. The reported cases differ are different because of the wall thermal flux, which is $63.05 \mathrm{~kW} / \mathrm{m}^{2}$ for Test $A$ and $204.91 \mathrm{~kW} / \mathrm{m}^{2}$ for Test B. The label "RNG" means RNG $k-\epsilon$ model and the label "SKE" means standard $k-\epsilon$ model. The reported markers are not representative of grid nodes.

fined within a small buffer region. In both tests the model of Bellmore and Reid underestimates the wall temperature $T_{w}<T_{w}^{e x p}$. This result partially contradicts the conclusion of Lee and Howell [36], which was probably due to previously discussed effects of discretization. The suggested approach for taking into account the effects of density fluctuations has been applied together with both the standard $k-\epsilon$ model and the RNG $k-\epsilon$ model. In both tests the standard $k-\epsilon$ model overestimates the wall temperature $T_{w}>T_{w}^{e x p}$. On the other hand, the RNG $k-\epsilon$ model produces the best results and this is even more true for the smallest wall thermal flux. This conclusion confirms one the peculiar feature of RNG $k-\epsilon$ model, which is to improve the near-wall description by taking into account the low-Reynolds-number effects [27]. The suggested approach allows to formulate numerical predictions of wall temperature which differ from experimental data by $\pm 20 \%$. This threshold allows to discriminate among different phenomenological correlations. It is interesting to note that experimental data are approximately midway-located into uncertainty range due to turbulent closure models. In order to complete the comparison of predicted profiles of dimensionless temperature with experi- 
Table 1

Comparison among numerical predictions of local heat transfer coefficients, experimental data of Wood and Smith [37] (label "W\&S") and other numerical predictions of Lee and Howell [36] (label "L\&H"). The considered models are: the model of Bellmore and Reid [24] (label "B\&R"); the RNG $k-\epsilon$ model (label "RNG") and the standard $k-\epsilon$ model (label "SKE"). The best results are bold-faced.

\begin{tabular}{cccccc}
\hline \hline & \multicolumn{4}{c}{ Test A: $q_{w}=63.05 \mathrm{~kW} / \mathrm{m}^{2}, T_{b}=302.82 K, R e=9.3 \times 10^{5}$} \\
\cline { 2 - 6 } & W\&S & L\&H & This Work & This Work & This Work \\
Parameters & (Exp.) & $(\mathrm{B} \& \mathrm{R})$ & $(\mathrm{B} \& \mathrm{R})$ & $(\mathrm{RNG})$ & $(\mathrm{SKE})$ \\
\hline$T_{w}[K]$ & 305.76 & 305.60 & 305.29 & 305.94 & 306.51 \\
$\alpha\left[k W / m^{2} K\right]$ & 21.45 & 23.88 & 25.53 & 20.21 & 17.09 \\
$e_{\alpha}[\%]$ & 0 & +11.3 & +19.0 & $-\mathbf{5 . 8}$ & -20.3 \\
\hline & Test B: & $q_{w}=204.91$ & $k W / m^{2}, T_{b}=303.15 K$, & $R e=9.3 \times 10^{5}$ \\
\cline { 2 - 6 } & W\&S & L\&H & This Work & This Work & This Work \\
Parameters & $($ Exp. $)$ & $(\mathrm{B} \& \mathrm{R})$ & $(\mathrm{B} \& \mathrm{R})$ & $(\mathrm{RNG})$ & $(\mathrm{SKE})$ \\
\hline$T_{w}[K]$ & 327.37 & 323.20 & 320.97 & 323.38 & 331.88 \\
$\alpha\left[k W / m^{2} K\right]$ & 8.46 & 10.62 & 11.50 & 10.13 & 7.13 \\
$e_{\alpha}[\%]$ & 0 & +25.5 & +35.9 & +19.7 & $-\mathbf{1 5 . 7}$ \\
\hline \hline
\end{tabular}

mental data of Wood and Smith [37], the whole radial profiles are reported into Fig. 5. The numerical predictions due to suggested approach are in good agreement with experimental data. In both tests the standard $k-\epsilon$ model reproduces better the dimensionless temperature profile in the bulk region. Despite the fact that in this case the local heat transfer coefficient is mainly due to wall temperature, this result suggests to consider in the following both the two-equation models, such as to better describe the turbulent diffusivities. This practice is confirmed by another reason. The final goal of this section is to estimate the local heat transfer coefficient $\alpha=q_{w} /\left(T_{w}-T_{b}\right)$, which is conventionally positive under heating conditions. If the wall thermal flux is imposed, then only the wall temperature $T_{w}$ depends on the calculation because the bulk temperature $T_{b}$ is unambiguously determinated by the inlet temperature $T_{0}$ and by the wall thermal flux $q_{w}$. This does not mean that the model, which reproduces better the wall temperature, will necessarily ensure the lowest error on local heat transfer coefficient. Let us introduce the numerical error on wall temperature as $e_{w}=\left(T_{w}^{e x p}-T_{w}\right) /\left(T_{w}^{e x p}-T_{b}\right)$ and the numerical error on local heat transfer coefficient as $e_{\alpha}=\left(\alpha-\alpha^{e x p}\right) / \alpha^{e x p}$. Since $\left|e_{w}\right|<<1$, then the numerical error on local heat transfer coefficient $e_{\alpha}$ can be developed near 
zero.

$$
e_{\alpha}=\frac{e_{w}}{1-e_{w}}=e_{w}+e_{w}^{2}+o\left(e_{w}^{2}\right)
$$

This means that the numerical models which overestimate the wall temperature, i.e. which are characterized by $e_{w}<0$, induce smaller errors on local heat transfer coefficient than the numerical models which underestimate it by the same absolute quantity $e_{w}>0$. This conclusion advantages the standard $k-\epsilon$ model because it usually overestimates the wall temperature. If the difference between performances of these models is small, then this conclusion must be taken into account to estimate local heat transfer coefficients. In Table 1 , the predicted values for both wall temperatures and local heat transfer coefficients are reported. For Test $B$, despite the fact that the RNG $k-\epsilon$ model works better to predict the wall temperature than the standard $k-\epsilon$ model $\left|e_{w}^{R N G}\right|<\left|e_{w}^{S K E}\right|$, the conclusion on local heat transfer coefficient is exactly turned off $\left|e_{\alpha}^{R N G}\right|>\left|e_{\alpha}^{S K E}\right|$. In the following calculation, both the RNG $k-\epsilon$ model and the standard $k-\epsilon$ model will be considered.

\subsection{Comparison with other predictions and experimental data for average heat transfer coefficient}

In this section, the discussed models will be adopted to predict the heat transfer under cooling conditions in mini/micro channels for carbon dioxide at supercritical pressure. The numerical results will be compared with some phenomenological correlations which allow to estimate the average heat transfer coefficients. Since these correlations generally produce conflicting predictions, it would be interesting to determine if the numerical results systematically show better agreement with a particular correlation. These calculations could help to settle the controversy on the existence of a heat transfer impairment in mini/micro channels in comparison with usual ducts. In all these calculations, the effects due to gravity field have not been intentionally included in order to verify if the buoyancy gives causes the heat transfer impairment. The basic problem is to decide what pattern of test conditions will best reveal aspects of investigated phenomena and will best allow a meaningful comparison with phenomenological correlations. This is a typical example of experimental design [38]. To perform a general experimental design, a fixed number of discrete values ("levels") for each of a number of variables ("factors") is selected and then all the possible combinations are experimentally considered. The goal of the experimental design is to characterize how the investigated quantity ("response") depends on considered factors. If there are $l_{1}$ levels for the first variable, $l_{2}$ for the second, $\ldots$ and $l_{k}$ for the $k$ th, the complete arrangement of $l_{1} \times l_{2} \times \cdots \times l_{k}$ experimental runs is called an $l_{1} \times l_{2} \times \cdots \times l_{k}$ factorial design. 
For the present application, there are five factors usually considered by all phenomenological correlations: the working pressure $p$; the bulk temperature $T_{b}(x)$; the wall temperature $T_{w}(x)$; the diameter of mini/micro channel $d$ and finally the mass flow rate $G$. In the experimental runs, the wall temperature $T_{w}(x)=T_{w}$ will be assumed uniformly distributed along axial direction and the final goal will be the calculation of the wall thermal flux $q_{w}(x)$. According to this strategy, the inlet bulk temperature $T_{b}(0)=T_{0}$ is the selectable factor. As previously discussed, the numerical prediction of turbulent convective heat transfer involves some proper hypotheses for describing turbulence: the turbulence model $M$ completes the set of factors. For the present application, the average heat transfer coefficient can be considered as the response. Unfortunately the definition of the average heat transfer coefficient $\alpha_{L}^{*}$ is not unique because it is essentially conventional. All the definitions share the same feature $\lim _{L \rightarrow 0} \alpha_{L}^{*}=\alpha$. In the present work, the following definition will be adopted:

$$
\alpha_{L}=\frac{\left(\int_{0}^{L} q_{w} d x\right) / L}{\left(\theta_{0}-\theta_{L}\right) / \ln \left(\theta_{0} / \theta_{L}\right)}
$$

where $\theta_{0}=T_{b}(0)-T_{w}$ and $\theta_{L}=T_{b}(L)-T_{w}$. The denominator of the previous expression should not trouble. The strong dependence of specific heat capacity on temperature near the critical point precludes the possibility to apply some elementary heat transfer models but it does not prevent to define a logarithmic mean temperature difference. The adopted average heat transfer coefficient $\alpha_{L}$ can not be directly compared with $\alpha_{L}^{*}$ due to the phenomenological correlations because the spurious comparison would be affected by both the experimental content and the differing definitions. In the present work, when a comparison between the numerical result and a phenomenological correlation is needed, the mini/micro channel is axially discretized into infinitesimal portions with length $d L$ and within each one the limit value $\alpha \approx \lim _{L \rightarrow d L} \alpha_{L}^{*}$ for local heat transfer coefficient is considered. Finally a coefficient coherent with definition (44) due to the phenomenological correlation is reconstructed. The major trends of the response can be usually investigated by means of only two levels. About the pressure, this means that a slightly supercritical pressure and a much higher pressure are considered. About the bulk temperature, the levels should allow to take into account the effects of pseudo-critical temperature but, in this case, two levels are not enough. Let us consider the following function:

$$
c_{p}^{w}(T)=\frac{1}{T-T_{w}} \int_{T_{w}}^{T} \bar{h}(T, p) d T
$$

This function allows to define the specific heat at wall conditions $c_{p}^{w w}=$ $\lim _{T \rightarrow T_{w}} c_{p}^{w}(T)$ and the average specific heat $c_{p}^{w b}=c_{p}^{w}\left(T_{b}\right)$. Many authors agree 
on the importance of the ratio $c_{p}^{w b} / c_{p}^{w w}$ between the average specific heat and the specific heat at wall conditions to characterize the heat transfer near the critical point $[9,13,15]$. Obviously this means that the heat transfer depends on the eventual presence of the pseudo-critical temperature in the range defined by wall and bulk conditions. Moreover this means that a configuration with the wall temperature close to the pseudo-critical value is not equivalent to a configuration with the bulk temperature close to the pseudo-critical value. These configurations are different because of the distortion of the radial temperature profile. Practically the ratio $c_{p}^{w b} / c_{p}^{w w}$ discriminates the cases characterized by $T_{b}(x) \approx T_{p c}$, which implies $c_{p}^{w b}>c_{p}^{w w}$, and the other cases characterized by $T_{w}(x) \approx T_{p c}$, which implies $c_{p}^{w b}<c_{p}^{w w}$. The factorial design must take into account both the configurations too. Three inlet bulk temperatures are selected: the first very close to pseudo-critical value $T_{0} \approx T_{p c}$; the second higher than previous one, so as that the wall temperature can be close to pseudo-critical value $T_{w} \approx T_{p c}$ and the third greatly higher. About the wall temperature, two levels are always considered and the difference $T_{0}-T_{w}$ is increased far from the pseudo-critical temperature where the heat transfer is weaker. Since the effects due to gravity field are neglected, the equation (1) allows to reduce the number of free parameters. The buoyancy parameter can be factorized $G r / R e_{0}^{2}=B o_{T} B o_{G}$. The first term $B o_{T}$ depends on the bulk and the wall temperatures, which have been previously selected, while the second term $\mathrm{Bo}_{G}$ depends on the mini/micro channel diameter and the mass flow rate.

$$
\begin{aligned}
& B o_{T}=\frac{\rho_{w} \rho_{0}-\rho_{0}^{2}}{\rho_{p c}^{2}} \\
& B o_{G}=\frac{\pi^{2}}{16} \frac{g \rho_{p c}^{2} d^{5}}{G^{2}}
\end{aligned}
$$

Since the selected factorial design implies $B o_{T} \leq 6.09 \times 10^{-1}$, the equation (1) reduces to $B o_{G} \leq 1.64 \times 10^{-3}$. In the following $B o_{G}=1.31 \times 10^{-3}$ is assumed and two levels for the mini/micro channel diameter $(d<2 \mathrm{~mm})$, or equivalently two levels for the mass flow rate, are selected. Finally three turbulence models are included: the approach of Bellmore and Reid and the suggested approach, together with both the RNG $k-\epsilon$ model and the standard $k-\epsilon$ model. The previous assumptions define a $2 \times 3 \times 2 \times 2 \times 3$ factorial design, which requires $2 \times 3 \times 2 \times 2 \times 3=72$ runs.

The numerical predictions of average heat transfer coefficient $\alpha_{L}$ are grouped by means of the selected levels for the supercritical pressure and for the diameter of mini/micro channel: in Table 2 the experimental runs $1-18$; in Table 3 the experimental runs $19-36$; in Table 4 the experimental runs $37-54$ and finally in Table 5 the experimental runs $55-72$. Before proceeding to compare these results with experimental data and other numerical predictions, a sensitivity analysis on considered factors is reported. About the turbulence 
Table 2

Numerical predictions of average heat transfer coefficient $\alpha_{L}$ for experimental runs 1-18 defined by the factorial design. The lowest supercritical pressure (7.412 $\mathrm{MPa})$ and the smallest mini/micro channel diameter $(0.787 \mathrm{~mm})$ are considered. The adopted models are: the model of Bellmore and Reid [24] (label "B\&R"); the RNG $k-\epsilon$ model (label "RNG") and the standard $k-\epsilon$ model (label "SKE"). The pseudo-critical temperature is $T_{p c}=304.328 \mathrm{~K}$.

\begin{tabular}{|c|c|c|c|c|c|c|c|c|c|}
\hline & \multicolumn{6}{|c|}{ Factorial Design } & \multicolumn{3}{|c|}{ Results } \\
\hline & $p$ & $T_{0}$ & $T_{w}$ & $d$ & $(G)$ & $M$ & $q_{w}$ & $\left|\Delta T_{b}\right|$ & $\alpha_{L}$ \\
\hline & {$[M P a]$} & {$[K]$} & {$[K]$} & {$[\mathrm{mm}]$} & {$[g / s]$} & & {$\left[k W / m^{2}\right]$} & {$[K]$} & {$\left[k W / m^{2} K\right]$} \\
\hline 1 & 7.412 & 305 & 302 & 0.787 & 0.571 & $\mathrm{~B} \& \mathrm{R}$ & 111.92 & 0.69 & 42.359 \\
\hline 2 & 7.412 & 305 & 302 & 0.787 & 0.571 & RNG & 115.57 & 0.69 & 43.803 \\
\hline 3 & 7.412 & 305 & 302 & 0.787 & 0.571 & SKE & 99.38 & 0.67 & 37.519 \\
\hline 4 & 7.412 & 305 & 298 & 0.787 & 0.571 & $\mathrm{~B} \& \mathrm{R}$ & 172.60 & 1.97 & 28.955 \\
\hline 5 & 7.412 & 305 & 298 & 0.787 & 0.571 & RNG & 176.55 & 2.19 & 30.244 \\
\hline 6 & 7.412 & 305 & 298 & 0.787 & 0.571 & SKE & 159.10 & 1.38 & 25.307 \\
\hline 7 & 7.412 & 312 & 309 & 0.787 & 0.571 & $\mathrm{~B} \& \mathrm{R}$ & 18.71 & 2.33 & 12.004 \\
\hline 8 & 7.412 & 312 & 309 & 0.787 & 0.571 & $\mathrm{RNG}$ & 17.52 & 2.19 & 10.484 \\
\hline 9 & 7.412 & 312 & 309 & 0.787 & 0.571 & SKE & 15.91 & 2.01 & 8.772 \\
\hline 10 & 7.412 & 312 & 305 & 0.787 & 0.571 & $\mathrm{~B} \& \mathrm{R}$ & 58.35 & 5.65 & 16.991 \\
\hline 11 & 7.412 & 312 & 305 & 0.787 & 0.571 & $\mathrm{RNG}$ & 54.49 & 5.41 & 14.972 \\
\hline 12 & 7.412 & 312 & 305 & 0.787 & 0.571 & SKE & 48.65 & 5.02 & 12.236 \\
\hline 13 & 7.412 & 360 & 353 & 0.787 & 0.571 & $\mathrm{~B} \& \mathrm{R}$ & 17.55 & 6.01 & 5.704 \\
\hline 14 & 7.412 & 360 & 353 & 0.787 & 0.571 & $\mathrm{RNG}$ & 16.76 & 5.74 & 5.013 \\
\hline 15 & 7.412 & 360 & 353 & 0.787 & 0.571 & SKE & 15.87 & 5.44 & 4.383 \\
\hline 16 & 7.412 & 360 & 340 & 0.787 & 0.571 & $B \& R$ & 50.23 & 16.56 & 5.344 \\
\hline 17 & 7.412 & 360 & 340 & 0.787 & 0.571 & RNG & 49.86 & 16.47 & 5.243 \\
\hline 18 & 7.412 & 360 & 340 & 0.787 & 0.571 & SKE & 47.12 & 15.60 & 4.575 \\
\hline
\end{tabular}


Table 3

Numerical predictions of average heat transfer coefficient $\alpha_{L}$ for experimental runs $19-36$ defined by the factorial design. The lowest supercritical pressure (7.412 $\mathrm{MPa})$ and the biggest mini/micro channel diameter $(1.417 \mathrm{~mm})$ are considered. The adopted models are: the model of Bellmore and Reid [24] (label "B\&R"); the RNG $k-\epsilon$ model (label "RNG") and the standard $k-\epsilon$ model (label "SKE"). The pseudo-critical temperature is $T_{p c}=304.328 \mathrm{~K}$.

\begin{tabular}{|c|c|c|c|c|c|c|c|c|c|}
\hline & \multicolumn{6}{|c|}{ Factorial Design } & \multicolumn{3}{|c|}{ Results } \\
\hline & $p$ & $T_{0}$ & $T_{w}$ & $d$ & $(G)$ & $M$ & $q_{w}$ & $\left|\Delta T_{b}\right|$ & $\alpha_{L}$ \\
\hline & {$[M P a]$} & {$[K]$} & {$[K]$} & {$[\mathrm{mm}]$} & {$[g / s]$} & & {$\left[k W / m^{2}\right]$} & {$[K]$} & {$\left[k W / m^{2} K\right]$} \\
\hline 19 & 7.412 & 305 & 302 & 1.417 & 2.482 & $\mathrm{~B} \& \mathrm{R}$ & 131.49 & 0.63 & 49.242 \\
\hline 20 & 7.412 & 305 & 302 & 1.417 & 2.482 & $\mathrm{RNG}$ & 162.26 & 0.66 & 61.020 \\
\hline 21 & 7.412 & 305 & 302 & 1.417 & 2.482 & SKE & 134.35 & 0.64 & 50.341 \\
\hline 22 & 7.412 & 305 & 298 & 1.417 & 2.482 & $\mathrm{~B} \& \mathrm{R}$ & 231.75 & 0.67 & 34.806 \\
\hline 23 & 7.412 & 305 & 298 & 1.417 & 2.482 & $\mathrm{RNG}$ & 280.40 & 0.70 & 42.188 \\
\hline 24 & 7.412 & 305 & 298 & 1.417 & 2.482 & SKE & 239.90 & 0.67 & 36.035 \\
\hline 25 & 7.412 & 312 & 309 & 1.417 & 2.482 & $\mathrm{~B} \& \mathrm{R}$ & 26.47 & 1.43 & 11.968 \\
\hline 26 & 7.412 & 312 & 309 & 1.417 & 2.482 & RNG & 26.22 & 1.41 & 11.813 \\
\hline 27 & 7.412 & 312 & 309 & 1.417 & 2.482 & SKE & 22.94 & 1.22 & 9.875 \\
\hline 28 & 7.412 & 312 & 305 & 1.417 & 2.482 & $\mathrm{~B} \& \mathrm{R}$ & 79.42 & 3.75 & 16.256 \\
\hline 29 & 7.412 & 312 & 305 & 1.417 & 2.482 & RNG & 80.34 & 3.79 & 16.522 \\
\hline 30 & 7.412 & 312 & 305 & 1.417 & 2.482 & SKE & 69.42 & 3.67 & 13.521 \\
\hline 31 & 7.412 & 360 & 353 & 1.417 & 2.482 & $B \& R$ & 27.84 & 3.98 & 5.879 \\
\hline 32 & 7.412 & 360 & 353 & 1.417 & 2.482 & RNG & 26.89 & 3.85 & 5.574 \\
\hline 33 & 7.412 & 360 & 353 & 1.417 & 2.482 & SKE & 24.43 & 3.50 & 4.838 \\
\hline 34 & 7.412 & 360 & 340 & 1.417 & 2.482 & $B \& R$ & 82.92 & 11.54 & 6.183 \\
\hline 35 & 7.412 & 360 & 340 & 1.417 & 2.482 & $\mathrm{RNG}$ & 79.99 & 11.15 & 5.850 \\
\hline 36 & 7.412 & 360 & 340 & 1.417 & 2.482 & SKE & 72.80 & 10.18 & 5.088 \\
\hline
\end{tabular}


Table 4

Numerical predictions of average heat transfer coefficient $\alpha_{L}$ for experimental runs $37-54$ defined by the factorial design. The highest supercritical pressure $(12.0 \mathrm{MPa})$ and the smallest mini/micro channel diameter $(0.787 \mathrm{~mm})$ are considered. The adopted models are: the model of Bellmore and Reid [24] (label "B\&R"); the RNG $k-\epsilon$ model (label "RNG") and the standard $k-\epsilon$ model (label "SKE"). The pseudo-critical temperature is $T_{p c}=327.1 \mathrm{~K}$.

\begin{tabular}{|c|c|c|c|c|c|c|c|c|c|}
\hline & \multicolumn{6}{|c|}{ Factorial Design } & \multicolumn{3}{|c|}{ Results } \\
\hline & $p$ & $T_{0}$ & $T_{w}$ & $d$ & $(G)$ & $M$ & $q_{w}$ & $\left|\Delta T_{b}\right|$ & $\alpha_{L}$ \\
\hline & {$[M P a]$} & {$[K]$} & {$[K]$} & {$[\mathrm{mm}]$} & {$[g / s]$} & & {$\left[k W / m^{2}\right]$} & {$[K]$} & {$\left[k W / m^{2} K\right]$} \\
\hline 37 & 12.000 & 327 & 317 & 0.787 & 0.571 & $\mathrm{~B} \& \mathrm{R}$ & 67.21 & 6.78 & 11.232 \\
\hline 38 & 12.000 & 327 & 317 & 0.787 & 0.571 & RNG & 70.36 & 7.13 & 12.322 \\
\hline 39 & 12.000 & 327 & 317 & 0.787 & 0.571 & SKE & 64.91 & 6.53 & 10.518 \\
\hline 40 & 12.000 & 327 & 307 & 0.787 & 0.571 & $\mathrm{~B} \& \mathrm{R}$ & 133.53 & 15.18 & 12.519 \\
\hline 41 & 12.000 & 327 & 307 & 0.787 & 0.571 & RNG & 132.12 & 14.98 & 12.189 \\
\hline 42 & 12.000 & 327 & 307 & 0.787 & 0.571 & SKE & 123.04 & 13.70 & 10.374 \\
\hline 43 & 12.000 & 347 & 337 & 0.787 & 0.571 & $\mathrm{~B} \& \mathrm{R}$ & 52.14 & 8.08 & 10.658 \\
\hline 44 & 12.000 & 347 & 337 & 0.787 & 0.571 & $\mathrm{RNG}$ & 49.65 & 7.75 & 9.549 \\
\hline 45 & 12.000 & 347 & 337 & 0.787 & 0.571 & SKE & 45.76 & 7.21 & 8.097 \\
\hline 46 & 12.000 & 347 & 327 & 0.787 & 0.571 & $\mathrm{~B} \& \mathrm{R}$ & 112.99 & 15.24 & 10.641 \\
\hline 47 & 12.000 & 347 & 327 & 0.787 & 0.571 & $\mathrm{RNG}$ & 118.00 & 15.75 & 11.606 \\
\hline 48 & 12.000 & 347 & 327 & 0.787 & 0.571 & SKE & 108.26 & 14.75 & 9.815 \\
\hline 49 & 12.000 & 360 & 353 & 0.787 & 0.571 & $\mathrm{~B} \& \mathrm{R}$ & 25.11 & 5.54 & 7.101 \\
\hline 50 & 12.000 & 360 & 353 & 0.787 & 0.571 & $\mathrm{RNG}$ & 25.22 & 5.56 & 7.176 \\
\hline 51 & 12.000 & 360 & 353 & 0.787 & 0.571 & SKE & 23.58 & 5.22 & 6.181 \\
\hline 52 & 12.000 & 360 & 340 & 0.787 & 0.571 & $B \& R$ & 81.61 & 16.01 & 8.214 \\
\hline 53 & 12.000 & 360 & 340 & 0.787 & 0.571 & RNG & 82.68 & 16.18 & 8.460 \\
\hline 54 & 12.000 & 360 & 340 & 0.787 & 0.571 & SKE & 76.88 & 15.23 & 7.237 \\
\hline
\end{tabular}


Table 5

Numerical predictions of average heat transfer coefficient $\alpha_{L}$ for experimental runs 55-72 defined by the factorial design. The highest supercritical pressure $(12.0 \mathrm{MPa})$ and the biggest mini/micro channel diameter $(1.417 \mathrm{~mm})$ are considered. The adopted models are: the model of Bellmore and Reid [24] (label "B\&R"); the RNG $k-\epsilon$ model (label "RNG") and the standard $k-\epsilon$ model (label "SKE"). The pseudo-critical temperature is $T_{p c}=327.1 \mathrm{~K}$.

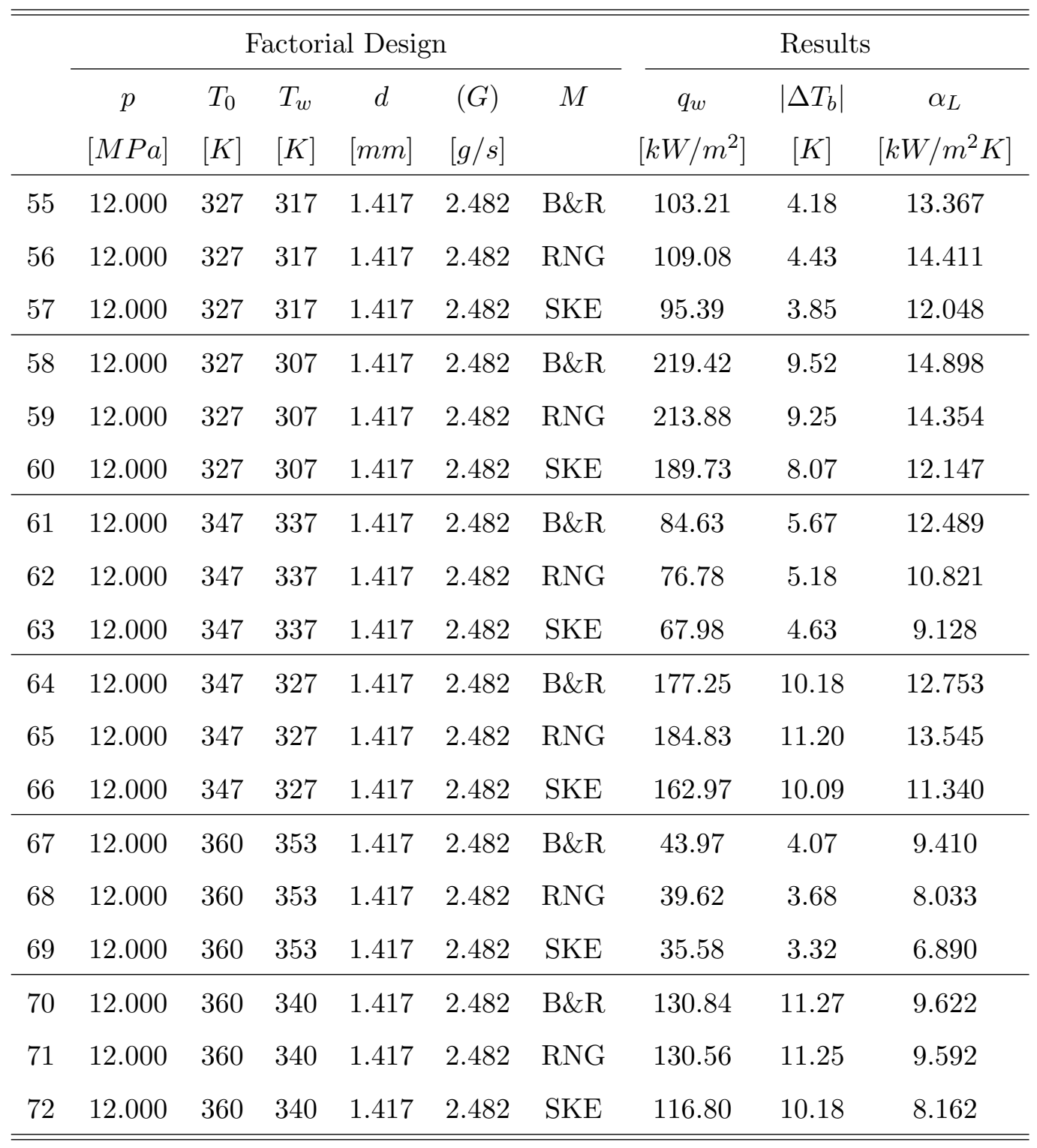



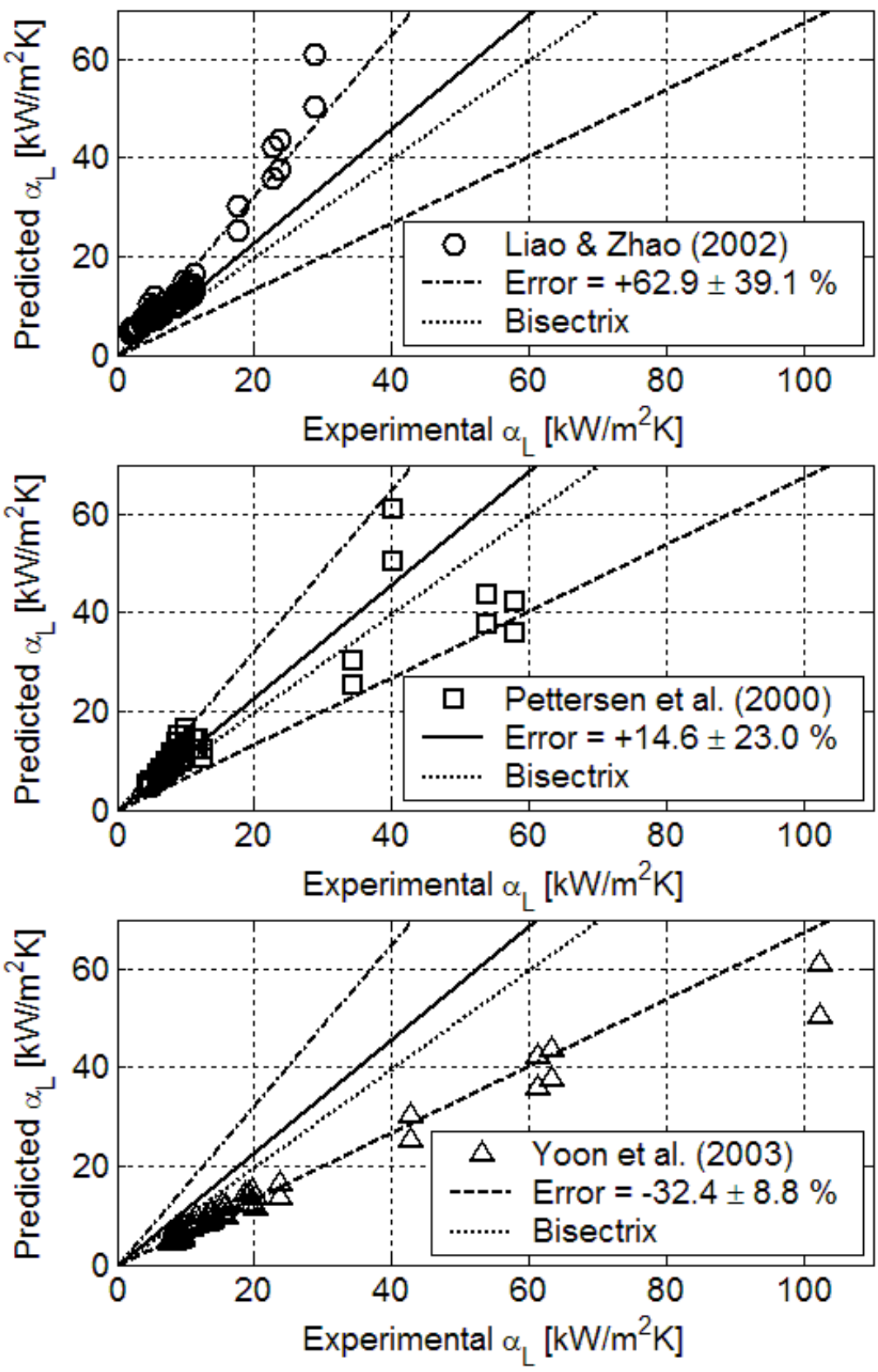

Fig. 6. The average heat transfer coefficients obtained by both the RNG $k-\epsilon$ model and the standard $k-\epsilon$ model are jointly reported, in order to duplicate the predictions for the same run. Some phenomenological correlations are considered $[13,11,15]$. For each subplot, the numerical error due to comparison with a phenomenological correlation is reported too, in terms of mean value and standard deviation. 
Table 6

Comparison among numerical predictions for average heat transfer coefficients, some phenomenological correlations $[13,11,12,15]$ and other numerical predictions [10]. The considered models are: the model of Bellmore and Reid [24] (label "B\&R"); the RNG $k-\epsilon$ model (label "RNG") and the standard $k-\epsilon$ model (label "SKE").

\begin{tabular}{ccccc}
\hline \hline & \multicolumn{4}{c}{ Numerical Predictions } \\
\cline { 2 - 5 } & \multicolumn{2}{c}{ Mean \pm Standard Deviation $e_{\alpha}^{L}=\left(\alpha_{L}-\alpha_{L}^{e x p}\right) / \alpha_{L}^{e x p}[\%]$} \\
\cline { 2 - 5 } Experimental & Petrov \& Popov & \multicolumn{3}{c}{ This work } \\
\cline { 2 - 5 } Correlations & Correlation & B\&R & RNG & SKE \\
\hline Liao \& Zhao & $50.6 \pm 34.6$ & $79.1 \pm 48.3$ & $76.2 \pm 39.2$ & $49.7 \pm 34.8$ \\
Pettersen et al. & $6.7 \pm 25.4$ & $25.9 \pm 27.1$ & $24.1 \pm 23.3$ & $5.1 \pm 18.6$ \\
Pitla et al. & $8.0 \pm 36.1$ & $25.5 \pm 23.7$ & $25.4 \pm 31.7$ & $6.3 \pm 25.9$ \\
Yoon et al. & $-37.6 \pm 6.2$ & $-25.8 \pm 11.8$ & $-26.8 \pm 7.4$ & $-37.9 \pm 6.2$ \\
\hline \hline
\end{tabular}

description, the standard $k-\epsilon$ model systematically produces lower values for average heat transfer coefficient in comparison with the RNG $k-\epsilon$ model. In the previous section, the fact that the standard $k-\epsilon$ model overestimates the effective temperature difference $\left|T_{b}-T_{w}\right|$ has been outlined yet and it is consistent with the present results. Moreover the numerical predictions due to the RNG $k-\epsilon$ model are rather close to the predictions due to the model of Bellmore and Reid. Usually the average heat transfer coefficients predicted by the RNG $k-\epsilon$ model are slightly lower than those due to the model of Bellmore and Reid, with the exception of the experimental runs which are characterized by $T_{0} \approx T_{p c}$ and which reveal a reverse trend. When the pseudo-critical temperature is close to bulk temperature, the radial temperature profile is highly distorted and it looks similar to a step function. Near the wall this feature implies a rapidly strained flow, which enhances the generation of turbulence kinetic energy. The additional terms, which are included in the RNG $k-\epsilon$ model to describe rapidly strained flows, probably justifies the increase in the predicted values for average heat transfer coefficient. About the inlet temperature difference $\left|T_{0}-T_{w}\right|$, the location of the pseudo-critical temperature plays an important part. The experimental runs 4 and 10 (see Table 2) share the same inlet temperature difference $\left|T_{0}-T_{w}\right|=7 \mathrm{~K}$ but for the first run $T_{b}(0) \approx T_{p c}$, which implies $c_{p}^{w b} / c_{p}^{w w}=3.94$, while for the second run $T_{w} \approx T_{p c}$, which implies $c_{p}^{w b} / c_{p}^{w w}=0.35$. The effect on the average heat transfer coefficient is impressive: $\alpha_{L}=28.96 \mathrm{~kW} / \mathrm{m}^{2} \mathrm{~K}$ for the experimental run 4 and $\alpha_{L}=16.99 \mathrm{~kW} / \mathrm{m}^{2} \mathrm{~K}$ for the experimental run 10 . This confirms the common practice to include the ratio $c_{p}^{w b} / c_{p}^{w w}$ in the phenomenological correlations and to assign it a positive exponent interpolating the experimental data. About 
the diameter of mini/micro channel, or equivalently the mass flow rate, the factorial design is based on the assumption to keep fixed the parameter $B o_{G}$, given by equation (47), so as to satisfy the threshold which allows to neglect the buoyancy effects. This means that $G^{2} / d^{5}$ is constant and then the inlet bulk velocity $u_{0} \propto G / d^{2} \propto d^{1 / 2}$ modestly increases by doubling the diameter of mini/micro channel. For this reason, the effects on the average heat transfer coefficients are relatively modest too. About the supercritical pressure, the peak of the specific heat at the pseudo-critical temperature enhances the convective heat transfer and the enhancement is proportional to the magnitude of the peak. The experimental runs 19 (see Table 3) and 55 (see Table 5) both are characterized by $T_{b}(0) \approx T_{p c}$, so that $c_{p}^{w b} / c_{p}^{w w}>1$. The effective temperature difference for the first experimental run $\left|T_{0}-T_{w}\right|=3 \mathrm{~K}$ is smaller than the one for the second experimental run $\left|T_{0}-T_{w}\right|=10 \mathrm{~K}$. In spite of this, the predicted wall thermal fluxes are comparable: $q_{w}=131.5 \mathrm{~kW} / \mathrm{m}^{2}$ for the experimental run 19 and $q_{w}=103.21 \mathrm{~kW} / \mathrm{m}^{2}$ for the experimental run 55. The reason lies in the magnitude of the peak of the specific heat at different supercritical pressures. The lowest supercritical pressure, considered by the first experimental run, causes the specific heat to strongly change in the radial direction $c_{p}^{w b} / c_{p}^{w w}=4.62$ while the highest supercritical pressure is much less effective $c_{p}^{w b} / c_{p}^{w w}=1.17$.

In Table 6 the numerical results are compared with other numerical predictions and some phenomenological correlations. The correlation suggested by Petrov and Popov [10] is included within the numerical results, because it was developed by interpolation of some numerical simulations. At least for the selected factorial design, this correlation reasonably agrees with the results due to the suggested approach for turbulence, if the standard $k-\epsilon$ model is adopted. This result highlights an interesting feature. The model of Bellmore and Reid, the model assumed by Petrov and Popov and finally the suggested approach differ from one another with reference to: how the variable thermophysical properties affect the common turbulent terms due to time averaging; how the turbulent terms can be calculated in terms of solving variables and how the the additional turbulent terms due to density fluctuations are described. In spite of this, the suggested approach reasonably reproduces both the previous models if the RNG $k-\epsilon$ model or the standard $k-\epsilon$ model are assumed. This means that the suggested approach is enough general to include different models independently developed. As it will clearer moreover, some of the additional topics included in this work produce moderate effects on the average heat transfer coefficients and this could hide additional discrepancies among the models. In Table 6, the experimental correlations due to Liao and Zhao [13], Pettersen et al. [11], Pitla et al. [12] and Yoon et al. [15] are considered too. These correlations are referable to three different categories. The first correlation was specifically developed for a single mini/micro channel. The second one derives from some experimental tests on a flat extruded tube, which involves many mini/micro channels along axial directions. The correlation of Pitla et al. improves the previous one by averaging the results obtained with 
constant properties evaluated at the wall and bulk temperature. Unfortunately this practice shifts the peak of the average heat transfer coefficient, which is no more located at the pseudo-critical temperature. This result is not confirmed by any theoretical explanation and it distinguishes this correlation from all other ones. Finally the correlation of Yoon et al. was recently developed for ducts with usual diameters. First of all, the numerical results seem to outline that the buoyancy effects are not completely responsible of the heat transfer impairment measured by Liao and Zhao for mini/micro channels. Despite the fact that the gravity field slightly affects the selected runs and it is completely neglected by numerical models, the final predictions systematically overestimate the results due to the correlation of Liao and Zhao. This conclusion is independent of turbulence model. If the experimental data are reliable, some additional terms must be included into the model to justify the heat transfer impairment for mini/micro channels. If a preference among phenomenological correlations on the basis of numerical results is needed, the results due to the RNG $k-\epsilon$ model and the standard $k-\epsilon$ model can be assembled. In Fig. 6 the assembled numerical results are compared with the phenomenological correlations. The assembled results seem to express a preference for the correlation suggested by Pettersen et al. [11]. This result is not conclusive because the experimental measurements for a single mini/micro channels should be more reliable than the measurements for a flat extruded tube, which can involve up to $25 \mathrm{mini} /$ micro channels and can be characterized by non-homogeneities for wall temperature. Anyway some numerical predictions show that the transverse non-homogeneities for a flat extruded tube are much more smaller than it could have been initially suppose [39].

Some concluding remarks on additional turbulent terms due to time averaging of density fluctuations are discussed. In Fig. 7 the corrective factor for turbulent diffusivities due to density fluctuations is reported. According to the assumed boundary conditions, the transverse sections of mini/micro channel closer to the inlet are characterized by stronger radial temperature gradients. This means they have higher indexes of intensity for density fluctuations $\sigma_{B R}$ and consequently more effective corrective factors for turbulent diffusivities $\phi_{B R}$. Nevertheless the maximum correction reported in Fig. 7 is less than $3 \%$. This threshold is even smaller for the suggested approach because $|\phi-1|<<\left|\phi_{B R}-1\right|$, as previously discussed. For the present application, this correction on turbulent diffusivities produces moderate effects on the average heat transfer coefficients and this prevents a complete comparison among discussed models. In Fig. 8 some results are reported for radial component of the characteristic velocity for density fluctuations, according to different models. The formal expression of radial component of the characteristic velocity for both the models is the same $\bar{v}^{*}=\bar{v}_{B R}^{*}$, as it can be easily verified by considering equations $(36,39)$. An estimation of the axial component of characteristic velocity can be obtained by means of the radial component for both the models, remembering that $\bar{u}^{*}<<\bar{u}_{B R}^{*}=\bar{v}_{B R}^{*}$. The RNG $k-\epsilon$ model overestimates the modulus of radial component of the characteristic velocity for the sections 


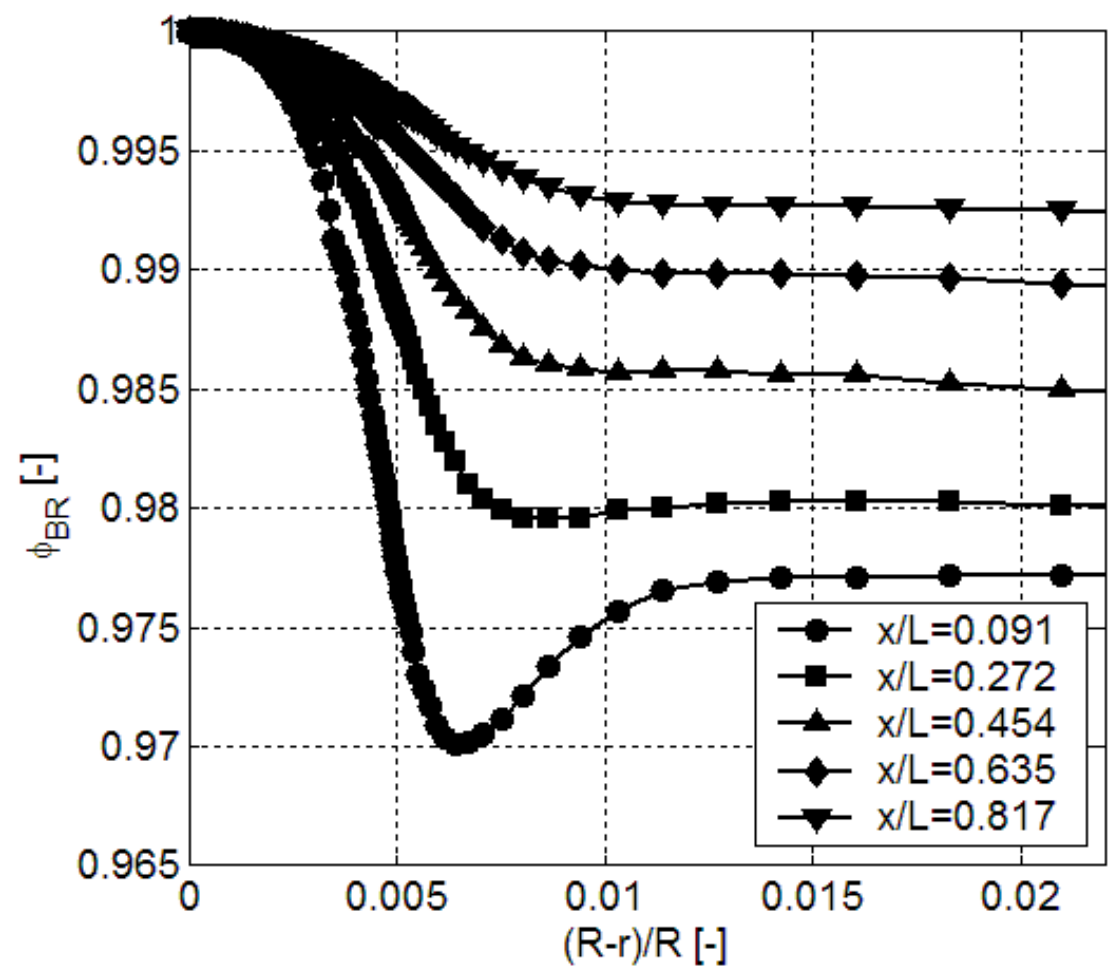

Fig. 7. Radial profile of the corrective factor for turbulent diffusivities due to density fluctuations in a thin layer near the wall, according to the model of Bellmore and Reid [24]. Cooling conditions are considered. The reported markers are representative of grid nodes.

closer to the inlet, while it underestimates the same quantity proceeding along the mini/micro channel. Also in this case, the additional terms, which are included in the RNG $k-\epsilon$ model to describe rapidly strained flows, probably justifies this increase in the radial component of the characteristic velocity for more distorted temperature profiles. Despite the fact that the density fluctuations strongly affect the radial component of the velocity $\bar{v} \approx-\bar{v}^{*}$, the final result on the average heat transfer coefficients is quite moderate.

\section{Conclusions}

A new approach to take into account the effects of variable physical properties on turbulence is suggested, by generalizing the decomposition originally considered by the model of Bellmore and Reid. This approach allows to choose freely the turbulent model for usual terms coming from time averaging of velocity fluctuations and to describe coherently the additional terms due to density fluctuations. In this way, the turbulence due to density fluctuations is analyzed under a general point of view, which imposes no constrain to the 


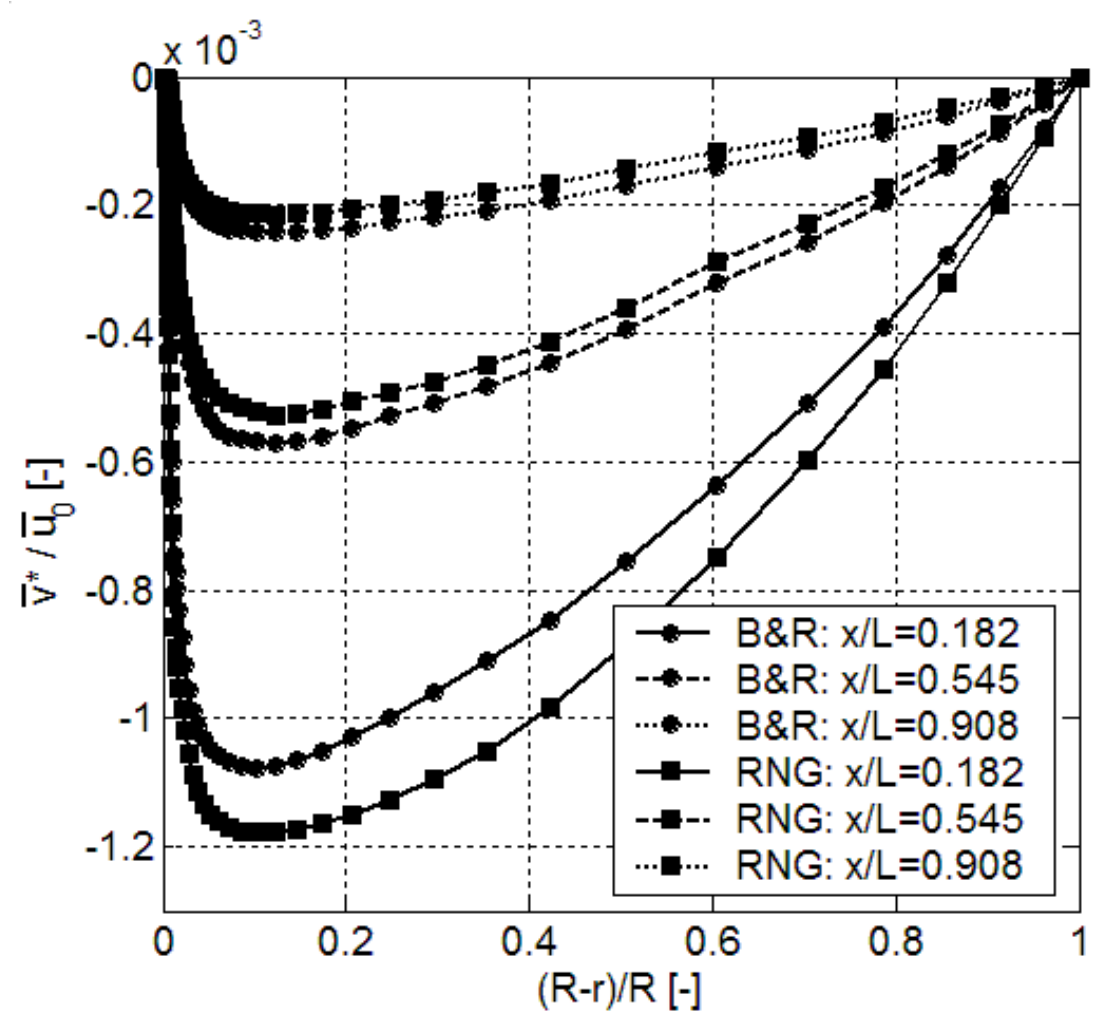

Fig. 8. Normalized radial component of the characteristic velocity for density fluctuations at the same locations, according to different models. The model of Bellmore and Reid (label "B\&R") and the suggested approach together with RNG $k-\epsilon$ model (label "RNG") are considered. The reported markers are representative of grid nodes.

description of usual terms. The suggested approach allows to reproduce some of the results due to some classical models, if the proper turbulent model is adopted.

Numerical calculations based on the suggested approach and on the original model have been performed for carbon dioxide flowing within mini/micro channels under cooling conditions. In comparison with existing calculations, some improvements have been considered: an updated database for thermophysical properties near the critical point; some differential equations to investigate the effects of variable thermophysical properties on turbulence; different turbulent closure models for usual terms and for additional terms due to density fluctuations. These refinements do not substantially improve the existing results. This means that for the considered application the effects due density fluctuations are smaller than it could have been initially supposed. The comparison with phenomenological correlations confirms that a heat transfer impairment for mini/micro channels exists but it is smaller than the impairment which has been measured by some experimental investigations for the same devices. The results are not completely exhaustive because of the discrepancies among different correlations. The strong coupling between the heat transfer and the 
fluid flow due to variable thermophysical properties complicates the development of a reliable correlation in terms of traditional dimensionless parameters. For this reason, some recent attempts [40] to adopt the neural network regression technique to analyze the convective heat transfer near the critical point appears greatly promising.

\section{Acknowledgments}

The author would like to acknowledge Prof. Michele Calì for creating the conditions for the development of the present work. This work was funded by Microtecnica s.r.l., a Hamilton Sundstrand company.

\section{A Appendix}

On introducing the Reynolds decomposition for velocity and density into instantaneous conservation equations and time-averaging the results, the governing equations of continuity (2), momentum (3) and energy (4) have been obtained. The density fluctuations affect both the diffusive and the convective terms into these equations. As previously pointed out, the corrective factor due to density fluctuations for effective diffusivities can be easily included into a segregated solver. On the other hand, the additional convective terms need a proper discretization. All the convection terms due to density fluctuations can be considered as additional sources into the previous equations and they can be recasted in the following generic form:

$$
H_{\omega}=-\nabla \cdot\left(\bar{\rho} \bar{\omega} \overline{\mathbf{w}}^{*}\right)=-\frac{\partial}{\partial x}\left(\bar{\rho} \bar{\omega} \bar{u}^{*}\right)-\frac{\partial}{\partial r}\left(\bar{\rho} \bar{\omega} \bar{v}^{*}\right)-\frac{\bar{\rho} \bar{\omega} \bar{v}^{*}}{r}
$$

where the quantity $\bar{\omega}$ can be indifferently $1, \bar{u}, \bar{v}$, or $\bar{h}_{T}$.

The generic source must be calculated by means of a conservative scheme, coherently with the finite volume method which has been selected. Let us consider a generic control volume, characterized by dimensions $\Delta x$ and $\Delta r$ along main directions. The centroid of the selected control volume is identified by $P(x, r)$ label. The centroids of the neighboring control volumes are identified by similar labels: $S\left(x, r-\Delta r_{S}\right), E\left(x+\Delta x_{E}, r\right), N\left(x, r+\Delta r_{N}\right)$ and $W\left(x-\Delta x_{W}, r\right)$. The midpoints of the faces of the selected control volume are identified by lower-case labels and progressive numbers: $s\left(x, r-\Delta r_{s}\right) \equiv 1$, $e\left(x+\Delta x_{e}, r\right) \equiv 2, n\left(x, r+\Delta r_{n}\right) \equiv 3$ and $w\left(x-\Delta x_{w}, r\right) \equiv 4$. The selected control volume is characterized by a volume equal to $\Delta V$. The generic $i$-th face of the selected control volumes is characterized by a surface equal to $\Delta A_{i}$. 
According to the introduced nomenclature, the divergence involved in the previous equation can be expressed:

$$
H_{\omega}=-\frac{\partial}{\partial V} \int_{\Delta V} \nabla \cdot\left(\bar{\rho} \bar{\omega} \overline{\mathbf{w}}^{*}\right) d V=-\frac{\partial}{\partial V} \int_{\partial \Delta V} \bar{\rho} \bar{\omega} \overline{\mathbf{w}}^{*} \cdot \mathbf{n} d A
$$

The continuous expression can be discretized:

$$
H_{\omega}=-\frac{1}{\Delta V} \sum_{i=1}^{4} \bar{\rho}_{i} \bar{\omega}_{i} \overline{\mathbf{w}}_{i}^{*} \cdot \mathbf{n}_{i} \Delta A_{i}
$$

This formula is conservative because it requires the estimation of the solving variables only at the boundary of the control volume. This feature ensures that the discretized equations for two adjoining control volumes involve the same flux through the common face. Since the calculation is performed only at the centroids of the control volumes, the solving variables at the midpoints of the faces can be estimated by means of linear interpolations. All terms of the summation can be explicitly reported:

$$
H_{\omega}=\bar{\rho}_{1} \bar{\omega}_{1} \bar{v}_{1}^{*} \frac{r-\Delta r_{s}}{r \Delta r}-\frac{\bar{\rho}_{2} \bar{\omega}_{2} \bar{u}_{2}^{*}}{\Delta x}-\bar{\rho}_{3} \bar{\omega}_{3} \bar{v}_{3}^{*} \frac{r+\Delta r_{n}}{r \Delta r}+\frac{\bar{\rho}_{4} \bar{\omega}_{4} \bar{u}_{4}^{*}}{\Delta x}
$$

They can be grouped:

$$
\begin{aligned}
H_{\omega}= & -\frac{\bar{\rho}_{2} \bar{\omega}_{2} \bar{u}_{2}^{*}-\bar{\rho}_{4} \bar{\omega}_{4} \bar{u}_{4}^{*}}{\Delta x}-\frac{\bar{\rho}_{3} \bar{\omega}_{3} \bar{v}_{3}^{*}-\bar{\rho}_{1} \bar{\omega}_{1} \bar{v}_{1}^{*}}{\Delta r} \\
& -\frac{\bar{\rho}_{3} \bar{\omega}_{3} \bar{v}_{3}^{*} \Delta r_{n}+\bar{\rho}_{1} \bar{\omega}_{1} \bar{v}_{1}^{*} \Delta r_{s}}{r\left(\Delta r_{s}+\Delta r_{n}\right)}
\end{aligned}
$$

The final expression is the discretized conservative form of the generic source. In the definition (A.1), the last term $\bar{\rho} \bar{\omega} \bar{v}^{*} / r$ is a peculiar feature of diverge operator when a set of cylindrical coordinates is considered. It can not be directly calculated by the values of solving variables at the boundary of the control volume. It is not evident how to calculate this term in discretized form, so as to ensure that the whole scheme is conservative. The previous formula (A.5) fills this lack by prescribing to use a linear interpolation of the volume-centered values along radial direction. It is interesting to note that the use of the centroid values would appear an easier solution but it would not produce a conservative scheme. 


\section{References}

[1] G. Lorentzen, J. Pettersen, A new efficient and environmentally benign system for car air-conditioning, International Journal of Refrigeration, 16 (1) (1993) $4-12$.

[2] J. Pettersen, A. Hafner, G. Skaugen, Development of compact heat exchangers for $\mathrm{CO}_{2}$ air-conditioning systems, International Journal of Refrigeration, 21 (3) (1998) 180-193.

[3] J. Millat, J.H. Dymond, C.A. Nieto de Castro, Transport properties of fluids, Cambridge University Press, Cambridge, United Kingdom, 1996.

[4] O.K. Rice, Critical phenomena, in: F.D. Rossini (Ed.), Thermodynamics and physics of matter, Princeton University Press, Princeton, New Jersey, 1955, pp. 419-499 (E).

[5] S. Kakaç, The effect of temperature-dependent fluid properties on convective heat transfer, in: S. Kakaç, R.K. Shah, W. Aung (Eds.), Handbook of singlephase convective heat transfer, Wiley, New York, 1987, pp. 18.1-18.56.

[6] B.S. Petukhov, Heat transfer and friction in turbulent pipe flow with variable physical properties, Advances in Heat Transfer, (6) (1970) 503-565.

[7] S.S. Pitla, D.M. Robinson, E.A. Groll, S. Ramadhyani, Heat transfer from supercritical carbon dioxide in tube flow: a critical review, International Journal of Heating Ventilating Air-Conditioning and Refrigerating Research, 3 (4) (1998) 281-301.

[8] E.A. Krasnoshchekov, I.V. Kuraeva, V.S. Protopopov, Local heat transfer of carbon dioxide at supercritical pressure under cooling conditions, High Temperature (translated from Teplofizika Vysokikh Tempeartur), 7 (5) (1970) 856-862.

[9] V.L. Baskov, I.V. Kuraeva, V.S. Protopopov, Heat transfer with the turbulent flow of a liquid at supercritical pressure in tubes under cooling conditions, High Temperature (translated from Teplofizika Vysokikh Tempeartur), 1 (15) (1977) 96-102.

[10] N.E. Petrov, V.N. Popov, Heat transfer and resistance of carbon dioxide being cooled in the supercritical region, Thermal Engineering (translated from Teploenergetika), 32 (3) (1985) 16-19.

[11] J. Pettersen, R. Rieberer, A. Leister, Heat transfer and pressure drop characteristics of supercritical carbon dioxide in microchannel tubes under cooling, International Institute of Refrigeration Commissions B1,B2,E1 and E2, Purdue University Press, 2000, pp. 99-106.

[12] S.V. Pitla, E.A. Groll, S. Ramadhayani, New correlation for the heat transfer coefficient during in-tube cooling of turbulent supercritical carbon dioxide, International Institute of Refrigeration Commissions B1,B2,E1 and E2, Purdue University Press, 2000, pp. 259-267. 
[13] S.M. Liao, T.S. Zhao, Measurements of heat transfer coefficients from supercritical carbon dioxide flowing in horizontal mini/micro channels, Journal of Heat Transfer, 124 (2002) 413-420.

[14] J.D. Jackson, W.B. Hall, Influences of buoyancy on heat transfer to fluids flowing in vertical tubes under turbulent conditions, Turbulent Forced Convection in Channels and Bundles, Hemisphere, New York, 1979.

[15] S.H. Yoon, J.H. Kim, Y.W. Hwang, M.S. Kim, K. Min, Y. Kim, Heat transfer and pressure drop characteristics during the in-tube cooling process of carbon dioxide in the supercritical region, International Journal of Refrigeration, 26 (2003) 857-864.

[16] V.N. Popov, N.E. Petrov, Calculation of heat transfer and flow resistance in the turbulent pipe flow of cooled carbon dioxide in the supercritical region, High Temperature (translated from Teplofizika Vysokikh Tempeartur), 23 (2) (1985) 309-316.

[17] V.N. Popov, E.P. Valueva, Heat transfer and turbulent flow of water at supercritical parameters if state in a vertical tube with significant effect of free convection, Thermal Engineering (translated from Teploenergetika), 33 (1986) $22-29$

[18] W.B. Hall, Heat transfer near the critical point, Advances in Heat Transfer, 7 (1981) 1-83.

[19] S.B. Pope, Turbulent flows, Cambridge University Press, Cambridge, 2000.

[20] H. Schlichting, Boundary layer theory, 4th edition, McGraw-Hill, New York, 1960.

[21] R.G. Deissler, C.S. Eian, Analytical and experimental investigation of fully developed turbulent flow of air in a smooth tube with heat transfer with variable fluid properties, NACA TN 2629, 1952.

[22] V.S. Sastry, N.M. Schnurr, An analytical investigation of forced convection heat transfer to fluids near the thermodynamic critical point, Journal of Heat Transfer, 97 (2) (1975) 226-230.

[23] K. Goldmann, Heat transfer to supercritical water and other fluids with temperature depedent properties, Chemical Engineering Progress Symposium, 50 (11) (1954) 105-113.

[24] C.P. Bellmore, R.L. Reid, Numerical prediction of wall temperatures for nearcritical para-hydrogen in turbulent upflow inside vertical tubes, Journal of Heat Transfer, 105 (1983) 536-541.

[25] D.C. Montgomery, G.C. Runger, N.F. Hubele, Engineering statistics, John Wiley \& Sons, New Jork, 2001.

[26] B.E. Launder, D.B. Spalding, Lectures in Mathematical Models of Turbulence, Academic Press, London, 1972. 
[27] H.K. Versteeg, W. Malalasekera, An introduction to computational fluid dynamics: the finite volume method, Addison-Wesley, London, 1996.

[28] V. Yakhot, S.A. Orszag, Renormalization group analysis of turbulence: I. basic theory, Journal of Scientific Computing, 1 (1) (1986) 1-51.

[29] M. Wolfstein, The velocity and temperature distribution of one-dimensional flow with turbulence augmentation and pressure gradient, International Journal of Heat and Mass Transfer, 12 (1969) 301-318.

[30] H.C. Chen, V.C. Patel, Near-wall turbulence models for complex flows including separation, AIAA Journal, 26 (6) (1988) 641-648.

[31] T. Jongen, Simulation and modeling of turbulent incompressible flows, PhD thesis, EPF Lausanne, Lausanne, Switzerland, 1992.

[32] V.V. Altunin, Thermophysical properties of carbon dioxide, Stardarty, Moscow, 1975 .

[33] E.W. Lemmon, M.O. McLinden, D.G. Friend, Thermophysical properties of fluid systems, in: P.J. Linstrom, W.G. Mallard (Eds.), NIST Chemistry WebBook, National Institute of Standards and Technology, 2003.

[34] J.H. Ferziger, M. Peric, Computational methods for fluid dynamics, SpringerVerlag, Berlin, 1996.

[35] S.V. Patankar, Numerical heat transfer and fluid flow, Hemisphere, New York, 1980.

[36] S.H. Lee, J.R. Howell, Turbulent developing convective heat transfer in a tube for fluids near the critical point, International Journal of Heat and Mass Transfer, 41 (10) (1998) 1205-1218.

[37] R.D. Wood, j.M. Smith, Heat transfer in the critical region - temperature and velocity profiles in turbulent flow, A.I.Ch.E. Journal, 10 (2) (1964) 180-186.

[38] G.E.P. Box, W.G. Hunter, J.S. Hunter, Statistics for experimentars, John Wiley \& Sons, New Jork, 1978.

[39] P. Asinari, L. Cecchinato, E. Fornasieri, Effects of Thermal Conduction in Microchannel Gas Coolers for Carbon Dioxide, International Journal of Refrigeration, (2004) Accepted for publication.

[40] G. Scalabrin, L. Piazza, M. Condosta, Convective cooling of supercritical carbon dioxide inside tubes: heat transfer analysis through neural network, International Journal of Heat and Mass Transfer, 46 (2003) 4413-4425. 